\title{
Using in-situ temperature measurements to estimate saturated soil thermal properties by solving a sequence of optimization problems
}

\author{
D. J. Nicolsky ${ }^{1}$, V. E. Romanovsky ${ }^{1}$, and G. S. Tipenko ${ }^{2}$ \\ ${ }^{1}$ Geophysical Institute, University of Alaska Fairbanks, PO Box 757320, Fairbanks, AK 99775, USA \\ ${ }^{2}$ Institute of Environmental Geoscience Russian Academy of Sciences, 13-2 Ulansky pereulok, PO Box 145, Moscow, Russia
}

Received: 1 August 2007 - Published in The Cryosphere Discuss.: 9 August 2007

Revised: 17 October 2007 - Accepted: 6 November 2007 - Published: 22 November 2007

\begin{abstract}
We describe an approach to find an initial approximation to the thermal properties of soil horizons. This technique approximates thermal conductivity, porosity, unfrozen water content curves in horizons where no direct temperature measurements are available. To determine physical properties of ground material, optimization-based inverse techniques are employed to fit the simulated temperatures to the measured ones. Two major ingredients of these techniques are an algorithm to compute the soil temperature dynamics and a procedure to find an initial approximation to the ground properties. In this article we show how to determine the initial approximation to the physical properties and present a new finite element discretization of the heat equation with phase change to calculate the temperature dynamics in soil. We successfully apply the proposed algorithm to recover the soil properties for the Happy Valley site in Alaska using oneyear temperature dynamics. The determined initial approximation is utilized to simulate the temperature dynamics over several consecutive years; the difference between simulated and measured temperatures lies within uncertainties of measurements.
\end{abstract}

\section{Introduction}

Recently, the Arctic Climate Impact Assessment report (ACIA, 2004) concluded that climate change is likely to significantly transform present natural environments, particularly across extensive areas in the Arctic and sub-Arctic. Among the highlighted potential transformations is soil warming which can potentially cause an increase in the active layer thickness and degradation of permafrost as well as have broader impacts on soil hydrology, northern ecosystems and infrastructure. Since permafrost is widely distributed and

Correspondence to: D. J. Nicolsky

(ftdjn@uaf.edu) covers approximately $25 \%$ of the land surface in the Northern Hemisphere (Brown et al., 1997), it is very important to understand the causes affecting soil temperature regime. One approach to studying soil temperature dynamics and their dependence on climate variability is to employ mathematical modeling (Goodrich, 1982; Nelson and Outcalt, 1987; Kane et al., 1991; Zhuang et al., 2001; Ling and Zhang, 2003; Oleson et al., 2004; Sazonova et al., 2004; Mölders and Romanovsky, 2006)

A mathematical model of soil freezing/thawing is based on finding a solution of a non-linear heat equation over a specified domain, (see Andersland and Anderson, 1978; Yershov, 1998, and many references therein). The domain represents ground material and is divided into several horizons (e.g. an organic matt, an organically enriched mineral soil layer, and a mineral soil layer) each with its distinct properties characterized by mineral-chemical composition, texture, porosity, heat capacity and thermal conductivity. By parameterizing the coefficients in the heat equation within each horizon, it is possible to take into account temperature-dependent latent heat effects occurring when ground freezes and thaws. This approach yields a realistic model of temperature dynamics in soils. However, in order to produce quantitatively reasonable results, it is necessary to prescribe physical properties of each horizon.

Conventional Time Domain Reflectometry (Topp et al., 1980) and drying methods are commonly used to estimate soil water content at shallow depths. The Time Domain Reflectometry method is based on measurements of the apparent dielectric constant around a wave guide inserted into the soil. It has been demonstrated that there is a relationship between the apparent dielectric constant and liquid water content (Topp et al., 1980) enabling robust estimations of water content in shallow soils with homogeneous composition. There are some difficulties however in measuring unfrozen water content of coarsely textured, heterogeneous or organically enriched soils in Arctic tundra (Boike and

Published by Copernicus Publications on behalf of the European Geosciences Union. 
Roth, 1997; Yoshikawa et al., 2004). More accurate measurements of the total water content (ice and water together) can be acquired by thermalization of neutrons and gamma ray attenuation. This is not always suitable for Arctic regions as it requires transportation of radioactive equipment to remote locations (Boike and Roth, 1997). An alternative to the above-mentioned methods and also to a number of others (Schmugge et al., 1980; Tice et al., 1982; Ulaby et al., 1982; Stafford, 1988; Smith and Tice, 1988) is the use of inverse modeling techniques. These techniques estimate the water content and other thermal properties of soil using in-situ temperature measurements and by exploiting the mathematical model.

A variety of inverse modeling techniques that recover the thermal properties of soil are known. Many of them rely on the commonly called source methods (Jaeger and Sass, 1964), in which temperature response due to heating is measured at a certain distance from the heat source. The temperature response and geometry of the probe are used to compute the thermal properties by either direct or indirect methods. In the direct methods, the temperature measurements are explicitly used to evaluate the thermal properties. In the indirect methods, one minimizes a discrepancy between the measured and the synthetic temperatures, the latter computed mathematically by exploiting the heat equation in which the coefficients are parameterized according to the specified thermal properties.

Application of direct methods such as the Simple Fourier Methods (Carson, 1963), Perturbed Fourier Method (Hurley and Wiltshire, 1993), and the Graphical Finite Difference Method (McGaw et al., 1978; Zhang and Osterkamp, 1995; Hinkel, 1997) yield accurate results for the thermal diffusivity (the ratio of the thermal conductivity and the heat capacity), only when water does not undergo the phase change. Despite the fact that the direct methods are well established for the heat equation without the phase change, no universal framework exists in the case of the soil freezing/thawing because the heat capacity and thermal conductivity depend strongly on the temperature in this case.

A common implementation of the indirect methods uses an analytical or numerical solution of the heat equation to evaluate the synthetic temperature. Due to strong non-linearities, the analytical solution of the heat equation is known only for a limited number of cases (Gupta, 2003), whereas numerical solutions are typically computable. Given a numerical solution computed by finite difference (Samarskii and Vabishchevich, 1996) or finite element (Zienkiewicz and Taylor, 1991) methods, one can minimize a cost function, $J$, which measures a discrepancy between the measured $T_{m}$ and synthetic $T_{c}$ temperatures. The typical expression for the cost function, $J$, is given by

$J(\mathcal{C}) \approx \int_{t_{s}}^{t_{e}}\left(T_{m}\left(x_{i}, t\right)-T_{c}\left(x_{i}, t ; \mathcal{C}\right)\right)^{2} d t$
Here, the quantity $\mathcal{C}$ is the control vector that is a set of parameters defining soil properties of each soil horizon. The synthetic temperature, $T_{c}$, is computed by the mathematical model parameterized by variables in $\mathrm{C}$ at some depths $x_{i}$ over the time interval $\left[t_{s}, t_{e}\right]$.

In this article, we deal with optimization techniques that find soil properties by minimizing the cost function (1). Commonly, the cost function $J$ is minimized iteratively starting from an initial approximation $\mathcal{C}_{0}$ to the parameters $\mathrm{C}$ (Thacker and Long, 1988). Since the heat equation is nonlinear, in general there are several local minima. Hence, it is important that the initial approximation lies in the basin of attraction of a proper minimum (Avriel, 2003).

We present a semi-heuristic algorithm to determine an initial approximation $\mathcal{C}_{0}$, for use as the starting point in multivariate minimization of cost functions such as (1). In this article, we use in-situ measured temperature $T_{m}$ to formulate the cost function $J$. We construct the initial approximation by minimizing cost functions over specifically selected time intervals $\left[t_{s}, t_{e}\right]$ in a certain order. For example, first, we propose to find thermal conductivity of the frozen soil using the temperature collected during winter, and then use these values to find properties of the thawed soil. In order to minimize the cost function it is necessary to compute the temperature dynamics multiple times for various control vectors $\mathcal{C}$. Since an analytical solution of the non-linear heat equation is not generally available, we use a finite element method to find its solution. To compute latent heat effects, we propose a new fixed grid technique to evaluate the latent heat terms in the mass (compliance) matrix using enthalpy formulation. Our techniques do not rely on temporal or spatial averaging of enthalpy, but rather evaluate integrals directly by employing a certain change of variables. An advantage of this approach is that it reduces the numerical oscillation of the temperature dynamics at locations near $0^{\circ} \mathrm{C}$ isotherm.

The structure of this article is as follows. In Sect. 2, we describe a commonly used mathematical model of temperature changes in the active layer and near surface permafrost. In Sect. 3, we outline a finite element discretization of the heat equation with phase change. In Sect. 4, we introduce main definitions, notations and state the variational approach to find the thermal properties. In Sect. 5, we provide an algorithm to construct an initial approximation to thermal properties. In Sect. 6, we apply our method to estimate the thermal properties and the coefficients determining the unfrozen water content at a site located in Alaska. In Sect. 7, we state limitations and shortcomings of the proposed algorithm. Finally, in Sect. 8, we provide conclusions and describe main results.

\section{Modeling of soil freezing and thawing}

For many practical applications, heat conduction is a dominant process, and hence the soil temperature $T,\left[{ }^{\circ} \mathrm{C}\right]$ can 
be simulated by a 1-D heat equation with phase change (Carslaw and Jaeger, 1959):

$C \frac{\partial}{\partial t} T(x, t)+L \frac{\partial}{\partial t} \theta_{l}(T, x)=\frac{\partial}{\partial x} \lambda \frac{\partial}{\partial x} T(x, t)$,

where $x \in[0, l], \quad t \in[0, \tau]$; the quantities $C=C(T, x)$ $\left[\mathrm{Jm}^{-3} \mathrm{~K}^{-1}\right]$ and $\lambda=\lambda(T, x)\left[\mathrm{Wm}^{-1} \mathrm{~K}^{-1}\right]$ stand for the volumetric heat capacity and thermal conductivity of soil, respectively; $L\left[\mathrm{Jm}^{-3}\right]$ is the volumetric latent heat of fusion of water, and $\theta_{l}$ is the volumetric liquid water content. We note that this equation is applicable when migration of water is negligible, there are no internal sources or sinks of heat, frost heave is insignificant, and there are no changes in topography and soil properties in lateral directions. Typically, the heat Eq. (2) is supplemented by Dirichlet, Neumann, or Robin boundary conditions specified at the ground surface, $x=0$, and at the depth $l$ (Carslaw and Jaeger, 1959). In geothermal studies, a Neumann boundary condition is typically set at the depth $l$. In this study we use the measured temperatures $T_{u}$ and $T_{l}$ to set the Dirichlet boundary conditions at depths $x=0$ and $x=l$, respectively, i.e. $T(0, t)=T_{u}(t), T(l, t)=T_{l}(t)$. In order to calculate the temperature dynamics $T(x, t)$ at any time $t \in[0, \tau]$, Eq. (2) is supplemented by an initial condition, i.e. $T(x, 0)=T_{0}(x)$, where $T_{0}(x)$ is the temperature at $x \in[0, l]$ at time $t=0$.

In certain conditions such as waterlogged Arctic lowlands, soil can be considered a porous media fully saturated with water. The fully saturated soil is a multi-component system consisting of soil particles, liquid water, and ice. It is known that the energy of the multi-component system is minimized when a thin film of liquid water (at temperature below $0^{\circ} \mathrm{C}$ ) separates ice from the soil particles (Hobbs, 1974). A film thickness depends on soil temperature, pressure, mineralogy, solute concentration and other factors (Hobbs, 1974). One of the commonly used measures of liquid water below freezing temperature is the volumetric unfrozen water content (Williams, 1967; Anderson and Morgenstern, 1973; Osterkamp and Romanovsky, 1997; Watanabe and Mizoguchi, 2002). It is defined as the ratio of liquid water volume in a representative soil domain at temperature $T$ to the volume of this representative domain and is denoted by $\theta_{l}(T)$. There are many approximations to $\theta_{l}$ in the fully saturated soil (Lunardini, 1987; Galushkin, 1997). The most common approximations are associated with power or exponential functions. Based on our positive experience in (Romanovsky and Osterkamp, 2000), we parameterize $\theta_{l}$ by a power function $\theta_{l}(T)=a|T|^{-b} ; a, b>0$ for $T<T_{*}<0^{\circ} \mathrm{C}$ (Lovell, 1957). The constant $T_{*}$ is called the freezing point depression (Hobbs, 1974), and from the physical point of view it means that ice does not exist in the soil if $T>T_{*}$. In thawed soils $\left(T>T_{*}\right)$, the amount of water in the saturated soil is equal to the soil porosity $\eta$, and hence the function $\theta_{l}(T)$ can be extended to $T>T_{*}$ as $\theta_{l}(T)=\eta$. Therefore, we assume that

$\theta_{l}(T, x)=\eta(x) \phi(T, x), \quad \phi=\left\{\begin{array}{ll}1, & T \geq T_{*} \\ \left|T_{*}\right|^{b}|T|^{-b}, & T<T_{*}\end{array}\right.$,

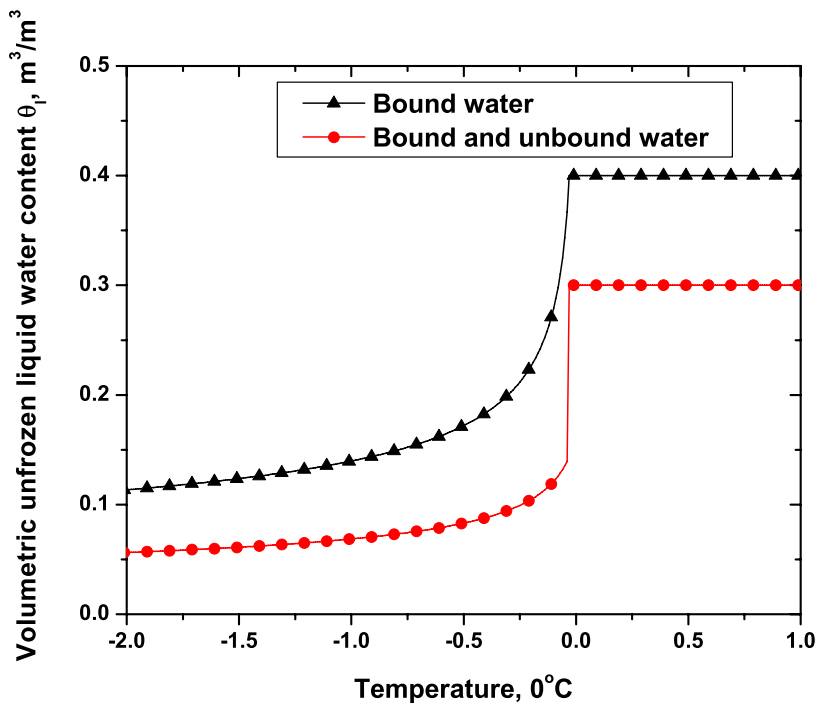

Fig. 1. Typical volumetric content of the unfrozen liquid water in soils as a function of temperature. The curve marked by triangles is associated with soils in which all water is bound in soil pores, and hence the water content gradually decreases with decreasing temperature in ${ }^{\circ} \mathrm{C}$. To compute this curve we used parametrization (3) in which $T_{*}=-0.03^{\circ} \mathrm{C}$ and $b=0.3$. The curve marked by circles is related to soils in which some percentage of water is not bound to the soil particle and changes its phase at the temperature $T_{*}$, while other part of liquid water is bound in soil pores and freezes gradually as the temperature decreases.

where $\phi=\phi(T, x)$ represents the liquid pore water fraction, and $T$ is in ${ }^{\circ} \mathrm{C}$, see the curve marked by triangles in Fig. 1 . Note that the constants $T_{*}$ and $b$ are the only parameters that specify dependence of the unfrozen liquid water content on temperature. For example, small values of $b$ describe the liquid water content in some fine-grained soils, whereas large values of $b$ are related to coarse-grained materials in which almost all water freezes at the temperature $T_{*}$. The limiting case in which all water freezes at the temperature $T_{*}$ is associated with phase change between water and ice (no soil particles). This limiting case is commonly called the classical Stefan problem and is represented by extremely large values of $b$ in (3).

In this article, we use the following notation and definitions. We abbreviate by letters $i, l$ and $s$, ice, liquid water, and the soil particles, respectively. We express thermal conductivity $\lambda$ of the soil and its apparent volumetric heat capacity $C_{\text {app }}$ according to (de Vries, 1963; Sass et al., 1971) as

$$
\begin{aligned}
& \lambda(T)=\lambda_{s}^{\theta_{s}} \lambda_{i}^{\theta_{i}(T)} \lambda_{l}^{\theta_{l}(T)}, \quad C_{\mathrm{app}}(T)=C(T)+L \frac{d \theta_{l}(T)}{d T} \\
& C(T)=\theta_{i}(T) C_{i}+\theta_{l}(T) C_{l}+\theta_{s} C_{s}
\end{aligned}
$$

where $C$ is called the volumetric heat capacity of the soil. Here, the constants $C_{k}, \lambda_{k}, k \in\{i, l, s\}$ are the volumetric heat 
Table 1. A typical thickness of soil layers and commonly occurring range of thermal properties in a cryosol soil at the North Slope, Alaska.

\begin{tabular}{lcccc}
\hline Layer & Layer thickness & $\begin{array}{c}\text { Thermal conductivity } \\
\text { in the frozen state, } \lambda_{f}\end{array}$ & $\begin{array}{c}\text { Porosity, } \\
\eta\end{array}$ & $\begin{array}{c}\text { Coefficient in (3) } \\
b\end{array}$ \\
\hline Moss or organic layer & 0.05 & {$[0.1,0.7]$} & {$[0.1,0.7]$} & {$[1.0,0.5]$} \\
Mineral-organic mixture & 0.20 & {$[0.9,1.6]$} & {$[0.2,0.6]$} & {$[0.8,0.5]$} \\
Mineral soil & $>1.0$ & {$[1.3,2.4]$} & {$[0.2,0.4]$} & {$[0.7,0.5]$} \\
\hline
\end{tabular}

capacity and thermal conductivity of the $k$-th constituent at $0^{\circ} \mathrm{C}$, respectively. The quantity $\theta_{k}, k \in\{i, l, s\}$ is the volume fraction of each constituent. Exploiting the relations $\theta_{s}=1-\eta$ and $\theta_{i}=\eta-\theta_{l}$, we introduce notation for the effective volumetric heat capacities $C_{f}$ and $C_{t}$, and the effective thermal conductivities $\lambda_{f}$ and $\lambda_{t}$ of soil for frozen and thawed states, respectively. Therefore formulae (4) and (5) yield

$C_{a p p}=C+L \frac{d \theta_{l}}{d T}, \quad C=C_{f}(1-\phi)+C_{t} \phi, \quad \lambda=\lambda_{f}^{1-\phi} \lambda_{t}^{\phi}$,

where

$\lambda_{f}=\lambda_{s}^{1-\eta} \lambda_{i}^{\eta}, \quad \lambda_{t}=\lambda_{s}^{1-\eta} \lambda_{l}^{\eta}$

$C_{f}=C_{s}(1-\eta)+C_{i} \eta, \quad C_{t}=C_{s}(1-\eta)+C_{l} \eta$

For most soils, seasonal deformation of the soil skeleton is negligible, and hence temporal variations in the total soil porosity $\eta$ for each layer are insignificant. Therefore, the thawed and frozen thermal conductivities for the fully saturated soil satisfy

$\frac{\lambda_{t}}{\lambda_{f}}=\left[\frac{\lambda_{l}}{\lambda_{i}}\right]^{\eta}$

It is important to emphasize that evaporation from the ground surface and from within the upper organic layer can cause partial saturation of upper soil horizons (Hinzman et al., 1991; Kane et al., 2001). Therefore, formula (7) need not hold within live vegetation and organic soil layers, and possibly within organically enriched mineral soil (Romanovsky and Osterkamp, 1997).

In this article, we approximate the coefficients $C_{\text {app }}, \lambda$ according to (6), where the thermal properties $\lambda_{f}, \lambda_{t}, C_{f}, C_{t}$ and parameters $\eta, T_{*}, b$ are constants within each soil horizon. Table 1 lists typical soil horizon geometry, commonly occurring ranges for the porosity $\eta$, thermal conductivity $\lambda_{f}$ and the values of $b$ parameterizing the unfrozen water content.

\section{Solution of the heat equation with phase change}

\subsection{A review of numerical methods}

In order to solve the inverse problem one needs to compute a series of direct problems, i.e. to obtain the temperature fields for various combinations of thermal properties. A number of numerical methods (Javierre et al., 2006) exist to compute temperature that satisfies the heat equation with phase change (2). These methods vary from the simplest ones which yield inaccurate results to sophisticated ones which produce accurate temperature distributions. The highly sophisticated methods explicitly track a region where the phase change occurs and produce a grid refinement in its vicinity, and therefore take significantly more computational time to obtain temperature dynamics. Implementing such complicated methods is not always necessary, since an extremely accurate solution is not particularly important when the mathematical model describing nature is significantly simplified.

In this subsection, we briefly review several fixed grid techniques (Voller and Swaminathan, 1990) that accurately estimate soil temperature dynamics and easily extend to multi-dimensional versions of the heat Eq. (2). These methods provide the solution for arbitrary temperature-dependent thermal properties of the soil and do not explicitly track the area where the phase change occurs. Recall that in soils the phase change occurs at almost all sub-zero temperatures. A cornerstone of the fixed grid techniques is a numerical approximation of the apparent heat capacity $C_{\text {app }}$. A variety of the approximation techniques can be found in (Voller and Swaminathan, 1990; Pham, 1995) and references therein. In general, two classes of them can be identified. The first class is based on temperature/coordinate averaging (Comini et al., 1974; Lemmon, 1979) of the phase change. Here, the apparent heat capacity is approximated by

$C_{\text {app }}=\frac{\partial H}{\partial x}\left(\frac{\partial T}{\partial x}\right)^{-1}$,

where

$H=\int_{0}^{T} C_{\text {app }} d T$,

is the enthalpy. The second class of methods is based on temperature/time averaging (Morgan et al., 1978). In this approach,

$C_{\text {app }}=\frac{H_{\text {current }}-H_{\text {previous }}}{T_{\text {current }}-T_{\text {previous }}}$,

where subscripts mark time steps at which the values of $H$ and $T$ are calculated. Although these methods have been presented in the context of large values of $b$ in (3), it is noted that 
they work best in the case of a naturally occurring wide phase change interval. Also, it is important to note that the approximation (8) is not accurate for near zero temperature gradients. In the case when the boundary conditions are given by natural variability (several seasonal freezing/thawing cycles), near zero gradients at some depths may occur for some time intervals. Hence, the temperature dynamics calculated by using (8) can have large computational errors.

An alternative fixed grid technique can be developed by rewriting the heat equation (2) in a new form:

$$
\frac{\partial H}{\partial t}=\frac{\partial}{\partial x} \lambda \frac{\partial}{\partial x} T, \quad T=T(H)
$$

resulting in the enthalpy diffusion method (Mundim and Fortes, 1979). Advantages of discretizing (10) is that the temperature $T=T(H)$ is a smooth function of enthalpy $H$ and hence one can compute all partial derivatives. However, for soils with a sharp boundary between thawed and completely frozen states, the enthalpy $H$ becomes a multivariate function when temperature $T$ nears $T_{*}$. Therefore, solution of (10) results in that the front becomes artificially stretched over at least one or even several finite elements.

In this article, we propose a fixed grid technique that applies the basic finite element method (Zienkiewicz and Taylor, 1991) to Eq. (2). Finite element discretization of

$$
L \frac{\partial \theta_{l}}{\partial t}=L \frac{d \theta_{l}}{d T} \frac{\partial T}{\partial t}
$$

in the left hand side of (2) results in

$$
\left(\int_{x_{0}}^{x_{1}} \psi_{i}(x) \psi_{j}(x) L \frac{d \theta_{l}}{d T}(T(x, t)) d x\right) \frac{d T_{j}}{d t},
$$

where $\psi_{i}(x)$ and $\psi_{i}(x)$ are two piecewise linear basis functions at nodes $i$ and $j$, respectively, $T_{j}(t)$ is the value of temperature at the $j$-th node at time $t$, and $T(x, t)=\sum_{i} \psi_{i}(x) T_{i}(t)$. We propose to evaluate this type of integrals using the unfrozen liquid water content $\theta_{l}$ as the integration variable, i.e.

$$
\int_{x_{0}}^{x_{1}} \psi(x) L \frac{d \theta_{l}}{d T}(T(x, t)) d x=L \int_{\theta_{0}}^{\theta_{1}} \psi\left(T\left(\theta_{l}, t\right)\right) d \theta_{l}
$$

where $\psi=\psi_{i} \psi_{j}$, and $\theta_{0}=\theta_{l}\left(T\left(x_{0}, t\right)\right)$ and $\theta_{1}=\theta_{l}\left(T\left(x_{1}, t\right)\right)$. This substitution allows precise computation of the latent heat effect for arbitrary grid cells, since it is parameterized by the limits of integration $\theta_{0}, \theta_{1}$, instead of being calculated using the rapidly varying function $\frac{d \theta_{l}}{d T}(T)$ on the element $\left[x_{0}, x_{1}\right]$ by a quadrature rule. As a consequence of the proposed substitution, evaluation of the integral in (11) may not to yield the right result unless the function $T\left(\theta_{l}\right)$ must be monotonically increasing for all $\theta_{l}<\eta$, and $T(x, t)$ be monotonous on $\left[x_{0}, x_{1}\right]$ at time $t$. Figure 1 shows two instances of the unfrozen water content curves frequently occurring in nature. The curve marked by circles is associated with soils in which free water freezes prior to freezing of the bound liquid water in soil pores. The free water is associated with a vertical line at $T=T_{*}$ whereas the bound water is represented by a smooth curve at $T<T_{*}$. The curve marked by triangles reflects soil in which all water is bounded in soil pores and can be parameterized by (3) used in our modeling.

\subsection{Finite element formulation}

Let us consider a triangulation of the interval $[0, l]$ by a set of nodes $\left\{x_{i}\right\}_{i=1}^{n}$. With each node $x_{i}$, we associate a continuous function $\psi_{i}(x)$ such that $\psi_{i}\left(x_{j}\right)=\delta_{i j}$. We will refer to $\left\{\psi_{i}\right\}_{i=1}^{n}$ as the basis functions on the interval $[0, l]$. Hence, the temperature $T(x, t)$ on $[0, l]$ is approximated by a linear combination: $T(x, t)=\sum_{i=1}^{n} T_{i}(t) \psi_{i}(x)$, where $T_{i}=T_{i}(t)$ is the temperature at the node $x_{i}$ at the time $t$. Substituting this linear combination into (2), multiplying it by $\psi_{j}$ and then integrating over the interval $[0, l]$, we obtain a system of differential equations (Zienkiewicz and Taylor, 1991):

$\mathbf{M}(\mathbf{T}) \frac{d}{d t} \mathbf{T}(t)=-\mathbf{K}(\mathbf{T}) \mathbf{T}(t)$,

where $\mathbf{T} \equiv \mathbf{T}(t)=\left[T_{1}(t) T_{2}(t) \ldots T_{n}(t)\right]^{t}$ is the vector of temperatures at nodes $\left\{x_{i}\right\}_{i=1}^{n}$ at time $t$. Here, the $n \times n$ matrices $\mathbf{M}(\mathbf{T})=\left\{m_{i j}\right\}_{i j=1}^{n}$ and $\mathbf{K}(\mathbf{T})=\left\{k_{i j}\right\}_{i j=1}^{n}$ are mass and stiffness matrices, respectively. Entry-wise they are defined as

$m_{i j}=\int_{0}^{l} C(T, x) \psi_{i} \psi_{j} d x+L \int_{0}^{l} \frac{d \theta_{l}}{d T} \psi_{i} \psi_{j} d x$
$k_{i j}=\int_{0}^{l} \lambda(T, x) \frac{d \psi_{i}}{d x} \frac{d \psi_{j}}{d x} d x$.

The fully implicit scheme is utilized to discretize (13) with respect to time. Denoting by $d t_{k}$ the time increment at the $k$-th moment of time $t_{k}$, one has

$\left[\mathbf{M}^{k}+d t_{k} \mathbf{K}^{k}\right] \mathbf{T}^{k}=\mathbf{M}^{k} \mathbf{T}^{k-1}, \quad k>1$

where $\mathbf{T}^{k}=\mathbf{T}\left(t_{k}\right), \mathbf{K}^{k}=\mathbf{K}\left(\mathbf{T}^{k}\right), \mathbf{M}^{k}=\mathbf{M}\left(\mathbf{T}^{k}\right)$. We impose boundary conditions at $x=0$ and some depth $x=l$ by specifying $T_{1}\left(t_{k}\right)=T_{u}\left(t_{k}\right)$ and $T_{n}\left(t_{k}\right)=T_{l}\left(t_{k}\right)$.

Given $\mathbf{T}^{k-1}$, we find the solution $\mathbf{T}^{k}$ of (16) by Picard iteration (Kolmogorov and Fomin, 1975). The iteration process starts from the initial guess $\mathbf{T}_{0}^{k}=\mathbf{T}^{k-1}$ that is used to compute temperature $\mathbf{T}_{1}^{k}$ at the first iteration. At iteration $s$, we compute $\mathbf{T}_{s}^{k}$ and terminate iterations at $s_{e}$ when a certain convergence condition is met. The value of $\mathbf{T}_{s}^{k}$ is used to evaluate the matrices $\mathbf{M}^{k}{ }_{s}=\mathbf{M}\left(\mathbf{T}_{s}^{k}\right)$, and $\mathbf{K}^{k}{ }_{s}=\mathbf{K}\left(\mathbf{T}_{s}^{k}\right)$. In turn, these are utilized to compute the $s+1$ iteration $\mathbf{T}_{s+1}^{k}$ by equating

$\left[\mathbf{M}^{k}{ }_{s}+d t_{k} \mathbf{K}^{k}{ }_{s}\right] \mathbf{T}^{k}{ }_{s+1}-\mathbf{M}^{k}{ }_{s} \mathbf{T}^{k-1}=0$.

At each iteration the convergence condition $\max _{k}\left|T_{k}^{s+1}\left(t_{k}\right)-T_{k}^{s}\left(t_{k}\right)\right| \leq \epsilon$ is checked. If it hold, the iterations are terminated at $s_{e}=s+1$. If the number of iterations exceeds a certain predefined number, the time increment $d t_{k}$ is halved and the iterations start again. Please, note that the convergence is guaranteed if the time increment $d t_{k}$ is small enough. 

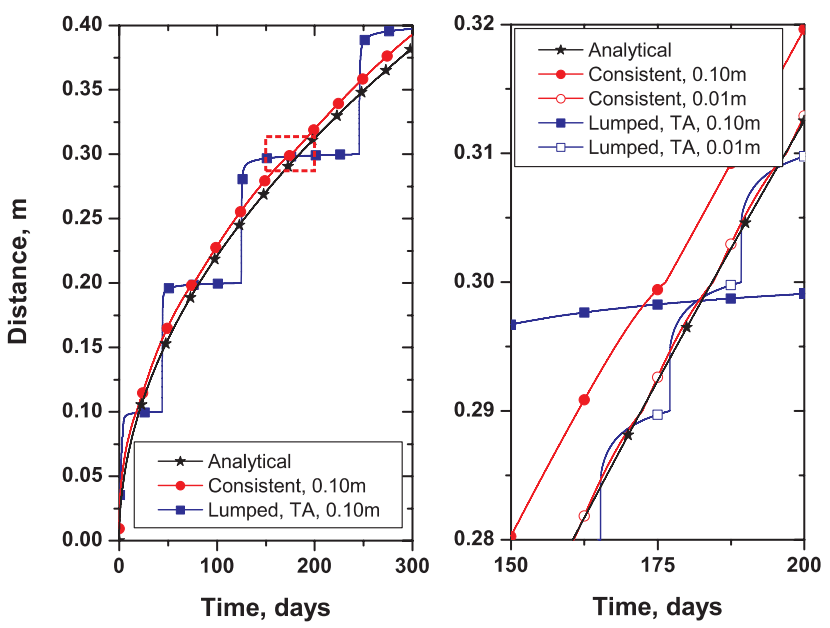

Fig. 2. Comparison of analytical (stars) and numerical solutions. Initially, the soil has $-5^{\circ} \mathrm{C}$ temperature, and at the time $t=0$, the temperature at its upper boundary is changed to $1^{\circ} \mathrm{C}$. At the lower boundary located at $5 \mathrm{~m}$ depth, zero flux boundary condition is specified. On the left plot, we show a location of the $0^{\circ} \mathrm{C}$ isotherm calculated for a uniform spatial discretizations with $0.1 \mathrm{~m}$ grid element. The numerical solutions are computed by the proposed method (circles) and by the scheme using the lumped approach with temporal enthalpy averaging (squares). In the right plot, we show an enlarged area within the dotted rectangle and a location of the $0^{\circ} \mathrm{C}$ isotherm calculated for a uniform spatial discretizations with $0.1 \mathrm{~m}$ (filled) and $0.01 \mathrm{~m}$ (hollow) grid elements.

\subsection{Computation of the mass matrix}

One of the obstacles to obtain a finite dimensional approximation that accurately captures the temperature dynamics is related to evaluation of the mass matrix M. Since the basis function $\psi_{i}$ does not vanish only on the interval $\left[x_{i-1}, x_{i+1}\right]$, the matrix $\mathbf{M}$ is tri-diagonal. Therefore, to compute its $i$-th row we evaluate

$\int_{0}^{l} \frac{d \theta_{l}}{d T} \psi_{j}(x) \psi_{i}(x) d x \quad j=i-1, i, i+1$,

where $j$ stands for the column index. For the sake of brevity, we consider the first integral $(j=i-1)$ in (18). This restricts us only to the grid element $\left[x_{i-1}, x_{i}\right]$, yielding

$\int_{0}^{l} \frac{d \theta_{l}}{d T} \psi_{i-1}(x) \psi_{i}(x) d x=\int_{x-1}^{x_{i}} \frac{d \theta_{l}}{d T} \psi_{i-1}(x) \psi_{i}(x) d x$.

We recall that in the standard finite element method, the temperature on the interval $\left[x_{i-1} x_{i}\right]$ is approximated by

$T(x, t)=\psi_{i-1}(x) T_{i-1}(t)+\psi_{i}(x) T_{i}(t)$,

for any $x \in\left[x_{i-1}, x_{i}\right]$ and fixed moment time $t$. Here, $\psi_{i}$ and $\psi_{i-1}$ are piece-wise linear functions satisfying $\psi_{i-1}=1-\psi_{i}$ on $\left[x_{i-1}, x_{i}\right]$. For all $x \in\left[x_{i-1}, x_{i}\right]$, we can compute the temperature $T$ from (20) and values of $T_{i}, T_{i-1}$. Note that in the case of $\Delta T_{i}=0$, we can compute (19) directly since $d \theta_{l} / d T$ is constant over $\left[x_{i-1} x_{i}\right]$. However, if $\Delta T_{i}=T_{i}-T_{i-1} \neq 0$, then we can consider an inverse function, that is, $x$ is taken as a function of $T$ to obtain

$\int_{x_{i-1}}^{x_{i}} \frac{d \theta_{l}}{d T} \psi_{i-1} \psi_{i} d x=\frac{x_{i}-x_{i-1}}{\left(\Delta T_{i}\right)^{3}} \int_{x_{i-1}}^{x_{i}} \frac{d \theta_{l}}{d T}\left(T_{i}-T\right)\left(T-T_{i-1}\right) d T$

Therefore

$\int_{0}^{l} \frac{d \theta_{l}}{d T} \psi_{i-1} \psi_{i} d x=\frac{x_{i}-x_{i-1}}{\left(\Delta T_{i}\right)^{3}} \int_{\theta_{i-1}}^{\theta_{i}}\left(T-T_{i}\right)\left(T_{i-1}-T\right) d \theta$,

where $\theta_{i-1}=\theta_{l}\left(T\left(x_{i-1}, t\right)\right)$ and $\theta_{i}=\theta_{l}\left(T\left(x_{i}, t\right)\right)$. Note that in (21) the temperature $T$ is a function of the liquid water content $\theta_{l}$, i.e. $T=\theta_{l}^{-1}\left(\theta_{l}\right)$. Therefore, returning back to (18), we have that each of the integrals in (18) is a linear combination of the type $\beta_{2} A_{2}+\beta_{1} A_{1}+\beta_{0} A_{0}$, where

$A_{k}=\int_{\theta_{i-1}}^{\theta_{i}}\left[\theta_{l}^{-1}(z)\right]^{k} d z, \quad k=0,1,2$.

The constants $\left\{\beta_{k}\right\}$ are easily computable if $\theta_{l}(T)$ is given by (3).

\subsection{Evaluation of the proposed method}

To test the proposed method, we compare temperature dynamics computed by the proposed method with an analytical solution of the heat Eq. (2) in which $b \rightarrow \infty$. This analytical solution is called Neumann solution (Gupta, 2003) and is typically used to verify numerical schemes. In the proposed numerical scheme the mass matrix $\mathbf{M}$ is tri-diagonal, and hence this scheme is called consistent. Other commonly utilized numerical schemes are called mass lumped (Zienkiewicz and Taylor, 1991) since they employ the diagonal mass matrix:

$\mathbf{M}=\operatorname{diag}\left(C_{\mathrm{app}, 1} \int_{0}^{1} \psi_{1} d x, \ldots, C_{\mathrm{app}, n} \int_{0}^{1} \psi_{n} d x\right)$.

Here, $C_{\mathrm{app}, i}$ is the value of the apparent heat capacity $C_{\mathrm{app}}$ at the $i$-th node computed either by spatial (8) or temporal (9) averaging of latent heat effects.

In Fig. 2, we compare temperature dynamics computed by the proposed consistent and a typical mass lumped scheme. We plot a location of the $0^{\circ} \mathrm{C}$ isotherm for several spatial discretizations, i.e. the distance $\Delta x_{i}$ between two neighboring nodes $x_{i}$ and $x_{i-1}$ is 0.1 or $0.01 \mathrm{~m}$. In this figure we see that the location of the $0^{\circ} \mathrm{C}$ isotherm calculated by numerical schemes lies within $\Delta x_{i}$ bound near the analytical solution. However, temporal dynamics of the location of the $0^{\circ} \mathrm{C}$ isotherm differ among methods. In the solution (squares) computed by the mass lumped approach with temporal enthalpy averaging (TA), dynamics of the $0^{\circ} \mathrm{C}$ isotherm has some irregularities, i.e. the freezing front either advancing too fast or too slow. In average, however this algorithm produces good results. Our proposed consistent method (circles) 


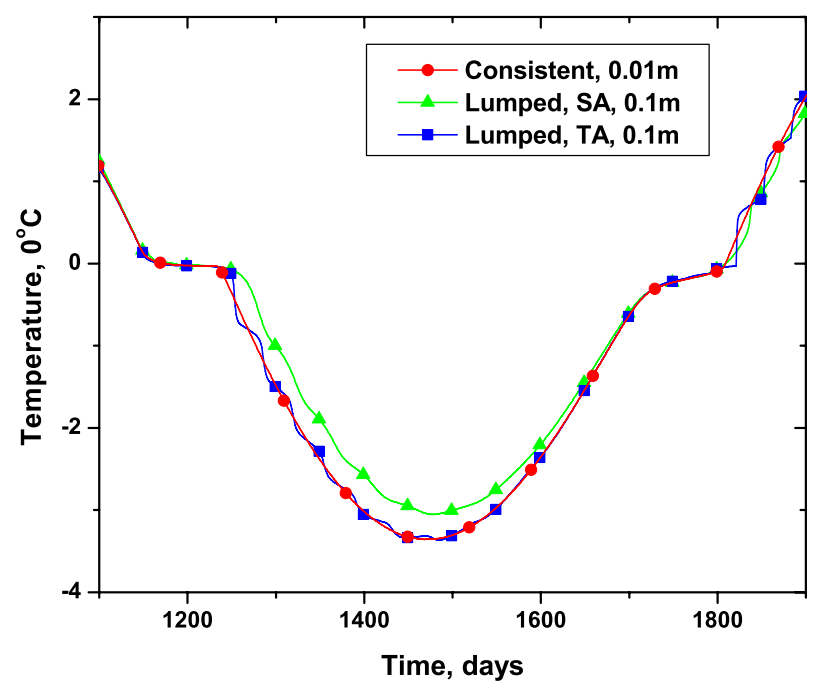

Fig. 3. Computed soil temperature dynamics at $0.3 \mathrm{~m}$ depth. Uniform 0.01 and $0.1 \mathrm{~m}$ meshes are used to compute temperatures by the consistent (circles) and mass lumped approaches, respectively. The spatial (SA) and temporal (TA) enthalpy averaging in lumped schemes are marked my triangles and squares, respectively. Initially the temperature is zero, the upper boundary condition is given by Dirichlet type boundary condition with a slowly varying sinusoid having the amplitude of $3^{\circ} \mathrm{C}$ and the period of there years; zero heat flux is specified at $2 \mathrm{~m}$ depth.

gives a better solution and smoother rate of advancing of the $0^{\circ} \mathrm{C}$ isotherm, see Fig. 2, left.

In Fig. 3, we compare temperature dynamics computed by two mass lumped approaches exploiting spatial (8) and temporal (9) enthalpy averaging. A warm bias in the temperature computed by the spatial averaging of the enthalpy is due to computational errors occurring when the temperature gradient is approximately zero at some depth. Our experience shows that this difference appears regardless of decreasing the tolerance $\epsilon$ between iterations in (17). We note that in all above numerical experiments a finite element computer code is the same except for a part associated with computation of mass matrix, i.e. consistent (18) or mass lumped (22). These numerical experiments show that the straight-forward mass lumped schemes are typically inferior to consistent ones.

Since our method (14) is based on the consistent approach (the mass matrix $\mathbf{M}$ is the tri-diagonal one), the numerical solution oscillates if the time steps $d t_{k}$ are too small (Pinder and Gray, 1977). For a fixed time step $d t_{k}$, the oscillations disappear if the spatial discretization becomes fine, i.e. the inequality $m_{i j}+d t_{k} k_{i j}<0$ holds when $i \neq j$ (Ciarlet, 2002; Dalhuijsen and Segal, 1986). It is shown that these oscillations occur due to violation of the discrete maximum principle (Rank et al., 1983). Therefore, to avoid the oscillations in the numerical solution (Dalhuijsen and Segal, 1986), we propose either to use sufficiently large time steps (for which

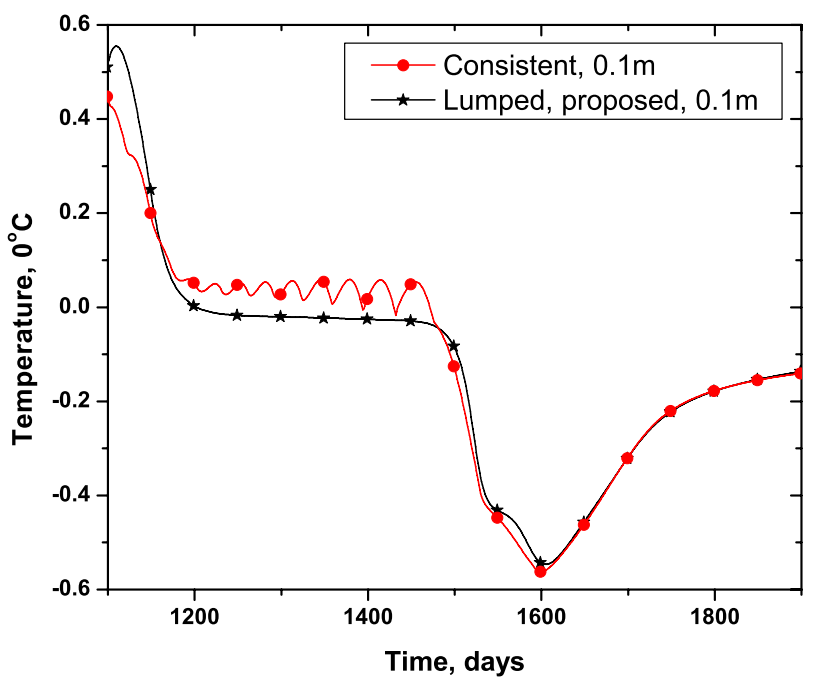

Fig. 4. Temperature dynamics at $1 \mathrm{~m}$ depth computed by the proposed consistent (circles) and the mass lumped schemes (stars). The mass lumped scheme is based on (23). In order to emphasis numerical oscillations occurring in the case of small time steps in the consistent approach, we use a uniform grid with $0.1 \mathrm{~m}$ grid elements. The oscillations are due to violation of the discrete maximum principle in the consistent scheme during active phase change processes. The initial and boundary conditions are the same as stated in caption of Fig. 3.

the formula can be found in the above cited references) or to exploit the following regularization. We construct a lumped version $\tilde{\mathbf{M}}=\left\{\tilde{m}_{i j}\right\}$ of the mass matrix $\mathbf{M}$ given by

$\tilde{m}_{i i}=\sum_{j} m_{i j}$

and substitute $\tilde{\mathbf{M}}$ for $\mathbf{M}$ in (16). Comparison of temperature dynamics computed employing the proposed consistent $\mathbf{M}$ defined by (16) and its mass lumped modification $\tilde{\mathbf{M}}$ defined by (23) is shown in Fig. 4. The numerical oscillations near $0^{\circ} \mathrm{C}$ disappear in the temperature dynamics computed by the proposed mass lumped approach (see Fig. 4). In Fig. 5, we compare the proposed mass lumped approach (stars), and the one based on temporal enthalpy averaging (squares) by (8). This figure shows that the numerical scheme using temporal averaging of the enthalpy produces larger oscillation than our solution. This comparison reveals that the proposed mass lumped approach (23) reduces some numerical oscillations and follows the "exact" solution (computed by the consistent approach with a fine spatial discretization) more closely than the solution computed by the lumped approach exploiting (8).

In conclusion, we state that if a spatial discretization is fine and time steps are sufficiently large (Pinder and Gray, 1977) then the consistent schemes do not show numerical oscillations, and hence they should be utilized. In the case of a 


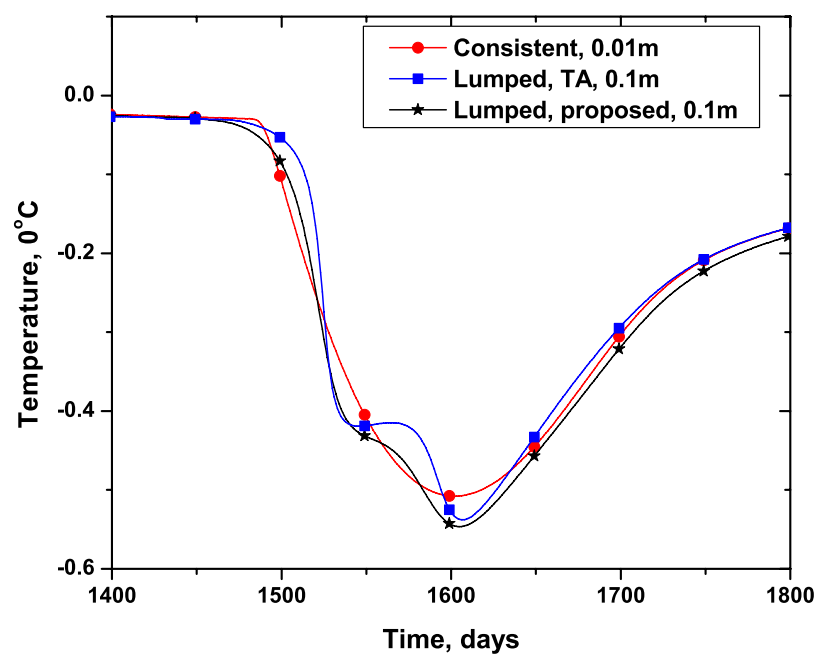

Fig. 5. Temperature dynamics at $1 \mathrm{~m}$ depth computed by the consistent approach (circles), the proposed mass lumped approach (stars) and the mass lumped approach with temporal enthalpy averaging (squares). The temperatures computed mass lumped approach are found on uniform grid with $0.1 \mathrm{~m}$ grid elements, whereas in the consistent approach, the length of grid elements is $0.01 \mathrm{~m}$. The initial and boundary conditions are the same as stated in caption of Fig. 3 .

coarse spatial discretization, consistent schemes can violate the discrete maximum principle, and hence the mass lumped schemes are more attractive. In this article, we construct a fine spatial discretization and use the proposed consistent approach, while restricting the time step $t_{k}$ from below.

\section{Variational approach to find the soil properties}

In this section, we provide definitions and describe main components of the indirect method used to find the soil properties by minimizing the cost function outlined in (1).

We define the control $\mathcal{C}$ as a set consisting of thermal conductivities $\lambda_{t}^{(i)}, \lambda_{f}^{(i)}$, heat capacities $C_{t}^{(i)}, C_{f}^{(i)}$ and parameters $\eta^{(i)}, T_{*}^{(i)}, b^{(i)}$ describing the unfrozen water content for each soil horizon $i=1, \ldots, n$, or

$\mathcal{C}=\left\{C_{f}^{(i)}, C_{t}^{(i)}, \lambda_{t}^{(i)}, \lambda_{f}^{(i)}, \eta^{(i)}, T_{*}^{(i)}, b^{(i)}\right\}_{i=1}^{n}$,

where $n$ is the total number of horizons. We say that a solution of the direct problem for the control $\mathcal{C}$ is $T(x, t ; \mathcal{C})$ and is defined by the set

$T(x, t ; \mathcal{C})=\left\{T\left(x_{i}, t\right): i=1, \ldots, m ; t \in[0, \tau]\right\}$,

where $\left\{x_{i}\right\}_{i=1}^{m}$ is a set of $m$ fixed distinct points on $[0, l]$. In (25), the $T\left(x_{i}, t\right)$ are point-wise values of temperature distributions satisfying (2) in which thermal properties of each horizon are given according to $\mathcal{C}$.

The counterpart of $T(x, t ; \mathcal{C})$ is the data $T_{\mathcal{D}}(x, t)$ defined by a set of measured temperature at the same depths $\left\{x_{i}\right\}_{i=1}^{m}$ and the same time interval $[0, \tau]$. Since the data $T_{\mathcal{D}}(x, t)$ and its model counterpart $T(x, t ; \mathcal{C})$ are given on the same set of depths and time interval, we can easily compute a discrepancy between them, usually measured by the cost function

$J(C)=\frac{1}{m\left(t_{s}-t_{e}\right)} \sum_{i=1}^{m} \frac{1}{\sigma_{i}^{2}} \int_{t_{s}}^{t_{e}}\left(T_{\mathcal{D}}\left(x_{i}, t\right)-T\left(x_{i}, t ; \mathcal{C}\right)\right)^{2} d t$.

Here, $t_{s}, t_{e} \in[0, \tau]$ and $\sigma_{i}$ stands for an uncertainty in measurements by the $i$-th sensor. In our measurements all temperature sensors assume the same precision, so all of $\left\{\sigma_{i}\right\}$ are equal. Given a way to measure this discrepancy as in (26) we can finally formulate an inverse problem.

For the given data $T_{\mathcal{D}}(x, t)$, we say that the control $\mathcal{C}_{*}$ is a solution to an inverse problem if discrepancy between the data and its model counterpart evaluated at $\mathcal{C}_{*}$ is minimal (Alifanov, 1995; Alifanov et al., 1996; Tikhonov et al., 1996). That is,

$J\left(\mathcal{C}_{*}\right)=\min _{\mathcal{C}} J(\mathcal{C})$.

To illustrate steps which are necessary to solve this inverse problem and find an optimal $\mathcal{C}_{*}$ we provide the following example. To formulate the inverse problem one has to have the measured temperatures $T_{\mathcal{D}}(x, t)$. For the sake of this example, we replace the data $T_{\mathcal{D}}(x, t)$ by a synthetic temperature $T_{\mathcal{S}}(x, t)=T\left(x, t ; \mathcal{C}^{\prime}\right)$ (a numerical solution of the heat Eq. (2) for the known combination $\mathcal{C}^{\prime}$ of the thermal properties):

$\mathrm{C}^{\prime}=\left\{\begin{array}{l}C_{f}^{(1)}=1.6 \times 10^{6}, C_{t}^{(1)}=2.1 \times 10^{6}, \lambda_{f}^{(1)}=0.55, \lambda_{t}^{(1)}=0.14, \eta^{(1)}=0.30, b^{(1)}=0.9, T_{*}^{(1)}=-0.03 \\ C_{f}^{(2)}=1.7 \times 10^{6}, C_{t}^{(2)}=2.3 \times 10^{6}, \lambda_{f}^{(2)}=\mathbf{0 . 9 0}, \lambda_{t}^{(2)}=0.66, \eta^{(2)}=0.30, b^{(2)}=0.6, T_{*}^{(2)}=-0.03 \\ C_{f}^{(3)}=1.8 \times 10^{6}, C_{t}^{(3)}=2.6 \times 10^{6}, \lambda_{f}^{(3)}=1.90, \lambda_{t}^{(3)}=1.25, \eta^{(3)}=\mathbf{0 . 2 5}, b^{(3)}=0.8, T_{*}^{(3)}=-0.03\end{array}\right\}$

The initial and boundary conditions in all calculations are fixed and given by in-situ temperature measurements in 2001 and 2002 at the Happy Valley site located in the Alaskan Arctic. We compute the temperature dynamics for a soil slab with dimensions $[0.02,1.06]$ between 21 July 2001 and 6 May 2002, and evaluate the cost function at $\left\{x_{i}\right\}_{i}=\{0.10$, $0.17,0.25,0.32,0.40,0.48,0.55,0.70,0.86 \mathrm{~m}$. Uniformly distributed noise on $[-0.04,0.04]$ was added to $T_{\mathcal{S}}(x, t)$, to simulate noisy temperature data recorded by sensors (precision of the sensor is $0.04^{\circ} \mathrm{C}$ ). The boundaries between the horizons lie at 0.10 and $0.20 \mathrm{~m}$ depth.

We find a control $\mathrm{C}^{\prime}$ that minimizes the cost function $J$ defined by (26) in which $T_{\mathcal{D}}(x, t)=T_{\mathcal{S}}(x, t)$. For the sake of simplicity, we assume that all variables in $\mathrm{C}^{\prime}$ are known except for the pair $\lambda_{f}^{(2)}, \eta^{(3)}$. Therefore, the problem of finding this pair can be solved by minimizing the cost function $J$ on $\left(\lambda_{f}^{(2)}, \eta^{(3)}\right)$ plane as follows. We compute temperature dynamics for various combinations of $\lambda_{f}^{(2)}, \eta^{(3)}$ and plot isolines of $J$, see Fig. 6 . The point on $\left(\lambda_{f}^{(2)}, \eta^{(3)}\right)$ plane where the cost function is minimal gives the sought values of $\lambda_{f}^{(2)}$ and $\eta^{(3)}$. The location of the minimum coincides with values $\lambda_{f}^{(2)}=0.9, \eta^{(3)}=0.25$, which were used to generate the synthetic data. 
In the above example, the control had only two unknown variables $\lambda_{f}^{(2)}, \eta^{(3)}$ and we minimized the corresponding cost function. Usually, a majority of variables in the control $\mathcal{C}$ is unknown, and hence multivariate minimization is required. Since computation of the cost function for all possible realizations of the control on the discrete grid is extremely timeconsuming, various iterative techniques are used (Fletcher, 2000).

We note that if the cost function has several minima due to non-linearities of the heat Eq. (2) and if the initial approximation $\mathcal{C}_{0}$ is arbitrary then the iterative algorithm can converge to an improper minimum. Nevertheless, with the initial approximation $\mathcal{C}_{0}$ within the basin of attraction of the global minimum, the iterative optimization method should converge to the proper minimum even if the model is nonlinear (Thacker, 1989). Consequently, proper determination of an initial approximation $\mathcal{C}_{0}$ is important.

After selection of the initial approximation $\mathcal{C}_{0}$, the next step is to minimize the cost function $J(\mathcal{C})$ with respect to all parameters in $\mathcal{C}$. There is a great variety of iterative methods that minimize $J(\mathcal{C})$. The majority of them rely on computation of the gradient $\nabla J(\mathcal{C})$ of the cost function. The computation of $\nabla J(\mathcal{C})$ is a complicated problem and is out of the scope of this article. An interested reader is referred to (Alifanov et al., 1996; Permyakov, 2004) and to references therein. Since in this article we are primarily concerned with evaluation of the initial approximation to the thermal properties, we use the following universal algorithm to minimize the cost function.

We look for the minimum of the cost function by the simplex search method described in (Lagarias et al., 1998), which is a direct search method (Bazaraa et al., 1993). In a two and three dimensional spaces, the simplex is a triangle or a pyramid, respectively. At each iteration the value of the function computed at the point, being in or near the current simplex, is compared with the function's values at the vertices of the simplex and, usually, one of the vertices is replaced by the new point, giving a new simplex. The iteration processes is continued until the simplex sizes are less than an a priori specified tolerance. At the final iteration, we obtain the set $\mathcal{C}$ of parameters that determine the thermal properties, porosity and coefficients specifying the unfrozen water content for each soil horizon. However, we note that this algorithm typically converges to the minimum slower than other algorithms that require calculations of the gradient (Dennis and Schnabel, 1987).

\section{Selection of an initial approximation}

Selection of a proper initial approximation $\bigodot_{0}$ is an important problem, since the proper choice of $\bigodot_{0}$ ensures that the minimization procedure converges to a global minimum. In this section we describe how to select a proper initial approximation by considering several simpler subproblems.

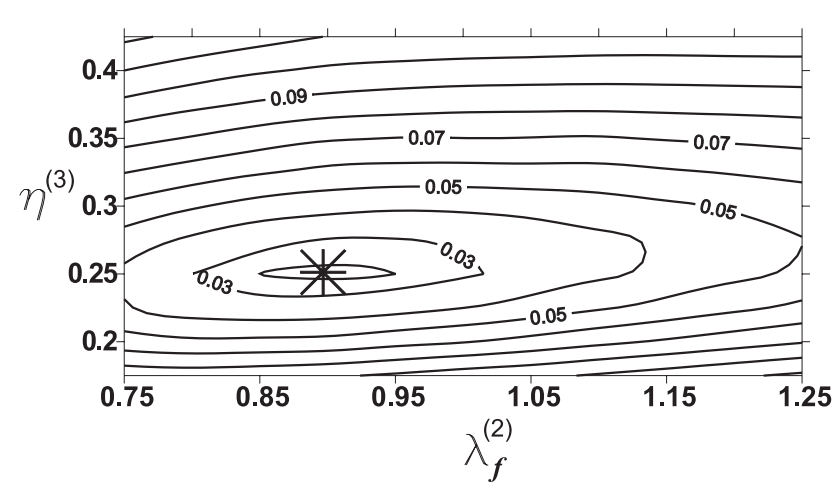

Fig. 6. Isolines of the cost function $J(\mathcal{C})$ computed using the synthetic temperature data $T_{\mathcal{S}}$. The minimum of the cost function is marked by the start and is located at $\lambda_{f}^{(2)}=0.9$ and $\eta^{(3)}=0.25$, which is coincide with the values of $\lambda_{f}^{(2)}, \eta^{(3)}$ used to compute $T_{\mathcal{S}}$.

\subsection{General methodology}

We begin by noting that in the natural environment, the thermal properties and the water content are confined within a certain range depending on soil texture and mineralogy. Therefore, the coefficients in (2) and hence their initial approximations lie within certain limits. To ensure better determination of the initial approximation $\mathcal{C}_{0}$, we employ an algorithm similar to coordinate-wise searching method (Bazaraa et al., 1993). In this method, one looks for a minimum along one coordinate, keeping other coordinates fixed, and then looks for the minimum along another coordinate keeping others fixed and so on.

We propose to look for a minimum with respect to some subset of parameters in $\mathcal{C}$, followed by a search along other parameters in $\mathcal{C}$ and so on. In details, our approach is formulated in five steps:

1. Select several time intervals $\left\{\Delta_{k}\right\}$ in the period of observations $[0, \tau]$

2. Associate a certain subset $\mathcal{C}_{j}$ of parameters $\mathcal{C}$ with each $\Delta_{j}$. The subset $\mathcal{C}_{j}$ is such that the temperature dynamics over the period $\Delta_{j}$ is primarily determined by $\mathcal{C}_{j}$ and depend much less on changes in any other parameters in $\mathrm{e}$.

3. Select a certain pair $\left\{\Delta_{j}, \mathcal{C}_{j}\right\}$, and look for a location of the minimum of the cost function $J(\mathcal{C})$ keeping all parameters in $\mathcal{C}$ except for $\mathcal{C}_{j}$ fixed.

4. Update values of $\mathcal{C}_{j}$ in the control $\mathcal{C}$ by the results obtained at Step 3.

5. Select another pair $\left\{\Delta_{i}, \mathcal{C}_{i}\right\}$ that is different from the pair $\left\{\Delta_{j}, \mathcal{C}_{j}\right\}$ at the previous step. Goto Step 3 and repeat for the pair $\left\{\Delta_{i}, \mathcal{C}_{i}\right\}$. 


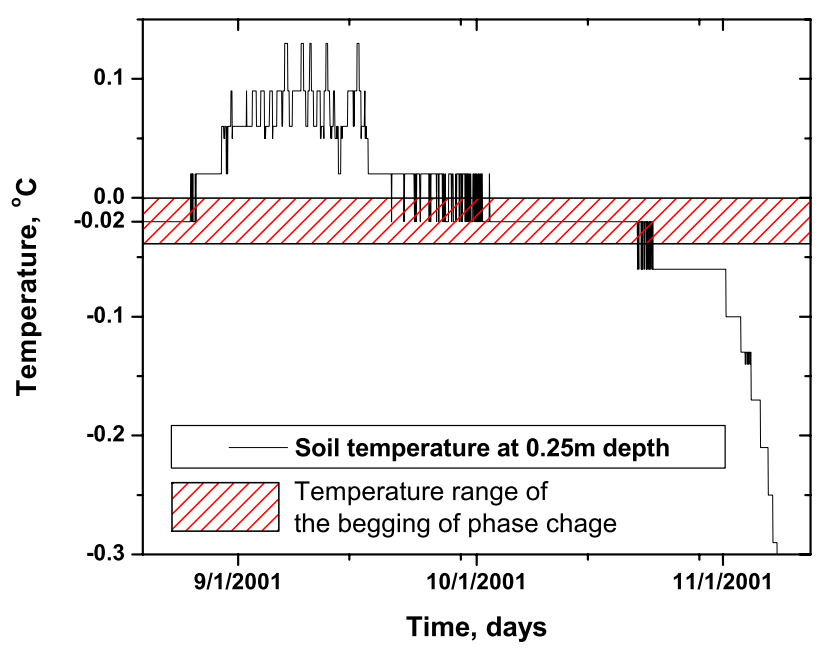

Fig. 7. Temperature dynamics at $0.25 \mathrm{~m}$ depth at Happy Valley site during the summer of 2001 year. The graph shows that uncertainty in temperature measurements is $\pm 0.02^{\circ} \mathrm{C}$. Within this uncertainty, the shadowed region represents a temperature range where the soil starts to freeze. Therefore, the temperature, $T_{*}$, of freezing point depression lies within the shadowed regions, i.e. in $\left[-0.04^{\circ} \mathrm{C} / 0^{\circ} \mathrm{C}\right]$.

We continue this iterative processes until the difference between the previous and current values of parameters in $\mathcal{C}$ is below a critical tolerance.

The selected periods $\Delta_{k}$ do not have to coincide with traditional subdivision of a year. The choice of $\Delta_{k}$ is naturally dictated by seasons in the hydrological year, which starts at the end of summer and consists several seasons. If the period of observations is one year, typical intervals $\Delta_{k}$ are "winter", "summer and fall", "fall" and "extended summer and fall", see Table 2. We note that the intervals $\Delta_{k}$ can overlap each other, and quantities $t_{s}$ and $t_{e}$ determining lower and upper limits of integration in (26) are equal to the beginning and end of the time interval $\Delta_{k}$. For different geographical regions, the timing for the "winter", "summer and fall" and "fall" can be different. Typical timing of periods $\left\{\Delta_{k}\right\}$ for the North Slope of Alaska is shown in Table 2, and are now discussed.

\subsection{Subproblems}

$\Delta_{1}$ : The "winter" period corresponds to the time when the rate of change of the unfrozen liquid water content $\theta_{l}$ is negligibly small; the heat Eq. (2) models the transient heat conduction with thermal properties $\lambda=\lambda_{f}, C=C_{f}$, and $\frac{d \theta_{l}}{d T} \simeq 0$. During the "winter", temperature dynamics depend only on the thermal diffusivity $C_{f} / \lambda_{f}$ of the frozen soil, and hence the simultaneous determination of both parameters $C_{f}$ and $\lambda_{f}$ is an ill-conditioned problem. Assuming that the heat capacity $\left\{C_{f}^{(i)}\right\}$ is known (depending on the soil texture and moisture content we can approximate it using published data), we evaluate the thermal conductivity $\left\{\lambda_{f}^{(i)}\right\}$ and use these values during minimization at other intervals.

$\Delta_{2}$ : During the "summer and fall" time interval, active phase change of soil moisture occurs. Hence, at this time, see Table 2, a contribution of the heat capacity $C$ into the apparent heat capacity $C_{\text {app }}$ is negligibly small comparing to the contribution of the latent heat term $L d \theta_{l} / d T$. Therefore, the rate of freezing/thawing primarily depends on the soil porosity $\eta$ and the thermal conductivity $\lambda$ (Tikhonov and Samarskii, 1963). Thus we approximate $\left\{C_{t}^{(i)}\right\}$ using published data by analyzing the soil texture and moisture content. Note that temperature-dependent latent heat effects due to the existence of unfrozen water $\theta_{l}$ at this period have a second order of magnitude effect (see discussion below). Therefore, if no prior information about the coefficients $b, T_{*}$ parameterizing the unfrozen water content is available then they can be prescribed by taking into account the soil texture and analyzing measured temperature dynamics at the beginning of freeze-up (see Fig. 7). We seek better estimates of $b, T_{*}$ at the next steps, namely during the "fall" period.

Since, during the "summer and fall" interval, the temperature dynamics primarily depends on the porosity $\eta$ and thermal conductivity $\lambda$, we have to find only $\left\{\lambda_{t}^{(j)}, \eta^{(j)}\right\}$, since $\left\{\lambda_{f}^{(j)}\right\}$ are already found at the previous step, i.e. the "winter" interval. Taking into account the relationship (7) between the thermal conductivities for completely frozen and thawed soil, we approximate

$\lambda_{t}^{(j)}=\lambda_{f}^{(j)}\left[\frac{\lambda_{l}}{\lambda_{i}}\right]^{\eta^{(j)}}, \quad j=2, \ldots, n$.

We remind that the water content $\theta_{l}$ in the upper soil horizon changes during the year due to moisture evaporation and precipitation and is not always equal to $\eta^{(1)}$. Hence formula (27) does not hold for $j=1$. Hence, during the "summer and fall" period our goal is to estimate $\left\{\eta^{(i)}\right\}_{i=1}^{n}$ and $\lambda_{t}^{(1)}$, and then determine thermal conductivity $\lambda_{t}^{(j)}$ for the rest of soil layers $j=2, \ldots, n$ using (27).

$\Delta_{3}$ : Recall that while evaluating the thermal properties $\left\{\lambda_{t}^{(i)}, \lambda_{f}^{(i)}\right\}$ and the soil porosity $\left\{\eta^{(i)}\right\}$, we assumed that the coefficients $\left\{b^{(i)}, T_{*}^{(i)}\right\}$ are known. However they also have to be determined. We remind that the coefficients $\left\{b^{(i)}, T_{*}^{(i)}\right\}$ cannot be computed prior to calculation of $\left\{\lambda_{t}^{(i)}, \lambda_{f}^{(i)}\right\}$ and $\left\{\eta^{(i)}\right\}$, since $\left\{b^{(i)}, T_{*}^{(i)}\right\}$ are related to the second order effects in temperature dynamics during "summer and fall" and "winter" intervals. Once an initial approximation to $\left\{\lambda_{t}^{(i)}, \lambda_{f}^{(i)}\right\}$ and $\left\{\eta^{(i)}\right\}$ is established, we consider the "fall" period (see Table 2) during which the temperature dynamics strongly depends on $\left\{b^{(i)}, T_{*}^{(i)}\right\}$ and allows us to capture second order effects in temperature dynamics (Osterkamp and Romanovsky, 1997).

$\Delta_{4}$ : In the previous three periods, we obtained approximations to all variables $\left\{\lambda_{t}^{(i)}, \lambda_{f}^{(i)}, C_{t}^{(i)}, C_{f}^{(i)}, \eta^{(i)}, b^{(i)}, T_{*}^{(i)}\right\}$. 
Table 2. Typical choice of parameters in the control $\varrho$ for "cold" permafrost regions.

\begin{tabular}{lllll}
\hline Periods & $\mathcal{C}_{j}$ & Typical $\Delta_{k}$ & Characteristic & Step \\
\hline "Winter" & $\left\{\lambda_{f}^{(i)}\right\}$ & December-April & Completely frozen ground, $T<-5^{\circ} \mathrm{C}$ & 1 \\
"Summer and Fall" & $\left\{\eta^{(i)}\right\}, \lambda_{t}^{(1)}$ & May-November & Developing/-ed active layer and its freezing & 2 \\
"Fall" & $\left\{b^{(i)}, T_{*}^{(i)}\right\}$ & September-December & Active layer freezing, $T>-5^{\circ} \mathrm{C}$ & 3 \\
"Extended Summer and Fall" & $\left\{\eta^{(i)}, T_{*}^{(i)}\right\}$ & May-January & Developing/-ed active layer and its freezing & 4 \\
\hline
\end{tabular}

However, we can improve the approximation by considering the "extended summer and fall" period, see Table 2. This period is associated with a time interval when the soil first thaws and then later becomes completely frozen. Since previously, we minimized the cost function depending separately on the porosity $\left\{\eta^{(i)}\right\}$ ("summer and fall") and on $\left\{T_{*}^{(i)}\right\}$ ("fall"), we minimize the cost function depending simultaneously on $\left\{\eta^{(i)}\right\}$ and $\left\{T_{*}^{(i)}\right\}$ during "extended summer and fall", while other parameters are fixed.

We list in Table 2 all steps and time periods $\Delta_{k}$ which are necessary to find the initial approximation. One of the sequences of minimization steps is

"winter" $\rightarrow$ "summer and fall" $\rightarrow$

"fall" $\rightarrow$ "extended summer and fall"

From our experience with this algorithm, we conclude that in some circumstances it is necessary to repeat minimization over some time periods several times, e.g.

"winter" $\rightarrow$ "summer and fall" $\rightarrow$

"fall" $\rightarrow$ "extended summer and fall" $\rightarrow$

"fall" $\rightarrow$ "extended summer and fall"

until the consecutive iterations modify the thermal properties insignificantly.

\section{Application. Happy Valley site}

\subsection{Short site description}

The temperature measurements were taken in the tussock tundra site located at the Happy Valley $\left(69^{\circ} 8^{\prime} \mathrm{N}, 148^{\circ} 50^{\prime} \mathrm{W}\right)$ in the northern foothills of the Brooks Range in Alaska from 22 July 2001 until 22 February 2005. We used data from 22 July 2001 until 15 May 2002 to estimate soil properties, and from 15 May 2002 until 22 February 2005 to validate the estimated properties. The site was instrumented by eleven thermistors arranged vertically at depths of $0.02,0.10,0.17$, $0.25,0.32,0.40,0.48,0.55,0.70,0.86$ and $1.06 \mathrm{~m}$. The temperature sensors were embedded into a plastic pipe (the MRC probe), that was inserted into a small diameter hole drilled into the ground. The empty space between the MRC and the ground was filled with a slurry of similar material to diminish an impact of the probe to the thermal regime of soil. Our frost heave measurements show that the vertical displacement of the ground versus the MRC probe is negligibly small at this particular installation site. Prior to the installation, all sensors were referenced to $0^{\circ} \mathrm{C}$ in an ice slush bath and have the precision of $0.04^{\circ} \mathrm{C}$. An automatic reading of temperature were taken every five minutes, then averaged hourly and stored in a data logger memory.

During the installation, soil horizons were described and their thicknesses were measured. The soil has three distinct horizons: organic cover, organically enriched mineral soil, and mineral soil. The boundaries between the horizons lie at 0.10 and $0.20 \mathrm{~m}$ depth.

In the all following numerical simulations we consider a slab of ground representing the Happy Valley soil between 0.02 and $1.06 \mathrm{~m}$ depth. For the computational purposes, the upper and lower boundary conditions are given by the observed temperatures at depth of 0.02 and $1.06 \mathrm{~m}$. Also in all computations, the temperatures are compared with the set of measured temperatures at the depths $\left\{x_{i}\right\}=\{0.10,0.17,0.25$, $0.32,0.40,0.48,0.55,0.70,0.86\} \mathrm{m}$.

\subsection{Selection of an initial approximation}

The "winter" period is associated to the ground temperature below $-5^{\circ} \mathrm{C}$, occurring on 15 January 2002 through 15 May 2002 at the Happy Valley site. The heat capacity $C_{f}$ for each layer is evaluated based on the soil type, texture and is taken from (Hinzman et al., 1991; Romanovsky and Osterkamp, 1995; Osterkamp and Romanovsky, 1996).

We estimate $\lambda_{f}$ for each layer by looking for a minimum of the cost function $J$ in the 3 -D space $\left\{\lambda_{f}^{(1)}, \lambda_{f}^{(2)}, \lambda_{f}^{(3)}\right\}$. The minimization problem in this space can be simplified by looking for a minimum in the following series of 2-D problems. For example, for several physically acceptable values of the thermal conductivity $\lambda_{f}^{(1)}$, we compute temperature dynamics for various values of $\lambda_{f}^{(2)}, \lambda_{f}^{(3)}$ and plot isolines of the cost function $J$. In the series of plots in Fig. 8, we notice that a location of the minimum on the $\left(\lambda_{f}^{(2)}, \lambda_{f}^{(3)}\right)$ plane shifts as $\lambda_{f}^{(1)}$ changes. The minimum of the cost function at each cross section is almost the same, and the problem of selecting the right combination of parameters arises. Here, knowledge of the soil structure becomes relevant. It is known that the soil type of third layer is silt highly enriched with ice, so from Table $11.6<\lambda_{f}^{(3)}<2.0$. Therefore, we select $\lambda_{f}^{(1)}=0.55$, $\lambda_{f}^{(2)}=1.0$ and $\lambda_{f}^{(3)}=1.8$, and use them in all other consecutive 

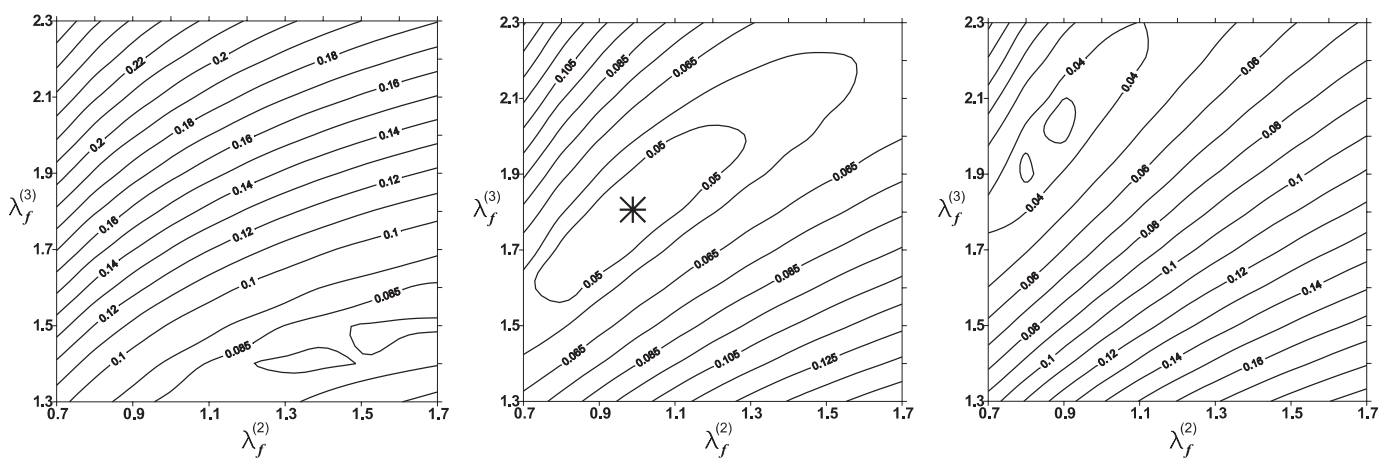

Fig. 8. The isolines of the cost function $J$ on the plane $\left(\lambda_{f}^{(2)}, \lambda_{f}^{(3)}\right)$ for different values of the thermal conductivity $\lambda_{f}^{(1)}$ keeping constant at each plot. The values of $\lambda_{f}^{(1)}$ from the left to the right are $0.35,0.55$ are 0.70 , respectively. The star in the central plot marks a selected combination of the thermal conductivities.

steps (see Table 3, columns 6,7 and 8). More precise results could be obtained if a sensor measuring the thermal conductivity was placed in at least one of the horizons, see discussion in Sect. 7.

The "summer and fall" period is selected to capture the maximal depth of active layer occurring between $28 \mathrm{Au}$ gust 2001 and 6 December 2001. We take values of the heat capacity $C_{t}$ from (Hinzman et al., 1991; Romanovsky and Osterkamp, 1995; Osterkamp and Romanovsky, 1996). Comparing measured temperatures to the ones computed for $\lambda_{t}^{(1)},\left\{\eta^{(i)}\right\}_{i=1}^{3}$ varying within a range of their natural variability, we found that $\lambda_{t}^{(1)} \in[0.09,0.15], \eta^{(1)} \in[0.3,0.9]$, $\eta^{(2)} \in[0.3,0.9]$ and $\eta^{(3)} \in[0.15,0.45]$. Once the variability of these parameters is found, we search for a minimum of the cost function in the 4-D space $\left\{\lambda_{t}^{(1)}, \eta^{(1)}, \eta^{(2)}, \eta^{(3)}\right\}$, where each parameter varies within the found boundaries. We note that during minimization of $J$ in this 4-D space, other variables in $\mathcal{C}$ are fixed and their values are listed in 1st "Summer and Fall" row in Table 3. For example, values of the thermal conductivity $\lambda_{f}^{(1)}=0.55, \lambda_{f}^{(2)}=1.0$ and $\lambda_{f}^{(3)}=1.8$ are obtained at the previous step after minimization over the "winter" interval. Also, an approximation to the coefficients $b^{(i)}=0.7$, $T_{*}^{(i)}=-0.03, i=1,2,3$ in (3) is obtained by analyzing soil texture and type, and dynamics of the measured temperatures near $0^{\circ} \mathrm{C}$, see Fig. 7. We emphasis that the approximation to the parameters $b$ and $T_{*}$ is tentative and is going to be improved at the consequent steps.

We note that it is not necessary to find a minimum in the four dimensional space accurately but rather only to estimate its location as significant uncertainties in other parameters still exist. Therefore, we look for the minimum by evaluating the cost function on $\left(\lambda_{t}^{(1)}, \eta^{(1)}\right),\left(\eta^{(1)}, \eta^{(2)}\right)$ and $\left(\eta^{(2)}, \eta^{(3)}\right)$ planes as follows.

First, we set $\eta^{(1)}=0.6, \eta^{(2)}=0.6, \eta^{(3)}=0.3$ and $\lambda_{t}^{(1)}=0.12$, which correspond to the middle of their variability ranges. Then, we evaluate the cost function $J$ on the $\left(\lambda_{t}^{(1)}, \eta^{(1)}\right)$ plane, by varying $\lambda_{t}^{(1)}, \eta^{(1)}$ in the control, while all other variables in $\mathcal{C}$ are fixed. In the left plot in Fig. 9, we plot isolines of $J$ on all three planes $\left(\lambda_{t}^{(1)}, \eta^{(1)}\right),\left(\eta^{(1)}, \eta^{(2)}\right)$, and $\left(\eta^{(2)}, \eta^{(3)}\right)$.

At the $\left(\lambda_{t}^{(1)}, \eta^{(1)}\right)$ plane, the cost function attains its minimal value on a boundary of this plane, see Fig. 9, upper left, and is minimal in the center of the planes $\left(\eta^{(1)}, \eta^{(2)}\right)$, $\left(\eta^{(2)}, \eta^{(3)}\right)$. The last two planes allows us to find that $\eta^{(1)}=0.6, \eta^{(2)}=0.55$ and $\eta^{(3)}=0.27$, whereas contours at the first plane show that the value of $\lambda_{t}^{(1)}$ lies between 0.11 and 0.13 , see Fig. 9, left column. We suppose that $\lambda_{t}^{(1)}$ is 0.12 and proceed further. After updating the control with the computed values, we evaluate the cost function on the same set of planes one more time; parameters in the control before minimization are shown in Table 3 the "Summer and Fall" 2nd row. After computing the cost function, we draw its isolines and show them in Fig. 9, right. Note that at this step the cost function attains its minima located in the center of the computational grid. We update the control with $\eta^{(1)}=0.6$, $\eta^{(2)}=0.55, \eta^{(3)}=0.27, \lambda_{t}^{(1)}=0.12$. Note that the location of the minimum did not change significantly. Our experience shows that changes of soil properties by $5 \%-10 \%$ or less are insignificant, since the corresponding difference in soil temperatures is comparable with uncertainties of measurements. Therefore, we do not have to do additional iterations on the same set of planes, and we proceed to the next step and reduce uncertainties in coefficients $T_{*}$ and $b$.

For the sake of brevity, we omit details in consequent steps associated with "fall" and "extended summer and fall" intervals, since the search of parameters is completed similar to as described in the "summer and fall" step. We emphasize that we are just interested in calculation of an initial approximation to the control which could serve as a starting point in the global minimization of the cost function. By no means, do we try to substitute the global minimization by this heuristic procedure. However, a good starting point can save computational time and improve accuracy of a final result. 
Table 3. Values of parameters in the control at the beginning of each minimization step. The listed steps are typical to recover the initial approximation to the soil properties. The parameters which values are in the parenthesis with the same subindex define minimization plane. For example, in the third row $\lambda_{t}^{(1)}$ and $\eta^{(1)}$ are in the parenthesis and have the same subindex equal to 1 . Therefore, this pair define a minimization plane $\left(\lambda_{t}^{(1)}, \eta^{(1)}\right)$. On this plane we minimize the cost function depending on $\lambda_{t}^{(1)}$ and $\eta^{(1)}$, while value of other parameters are fixed and given in other sections of the current row.

\begin{tabular}{|c|c|c|c|c|c|c|c|c|c|c|c|c|c|}
\hline Iterations & $\eta^{(1)}$ & $\eta^{(2)}$ & $\eta^{(3)}$ & $\lambda_{t}^{(1)}$ & $\lambda_{f}^{(1)}$ & $\lambda_{f}^{(2)}$ & $\lambda_{f}^{(3)}$ & $b^{(1)}$ & $b^{(2)}$ & $b^{(3)}$ & $T_{*}^{(1)}$ & $T_{*}^{(2)}$ & $T_{*}^{(3)}$ \\
\hline Winter & 0.40 & 0.70 & 0.25 & 0.10 & - & - & - & 0.7 & 0.7 & 0.7 & -0.03 & -0.03 & -0.03 \\
\hline Summer and Fall, 1st & $(0.60)_{1,2}$ & $(0.60)_{2,3}$ & $(0.30)_{3}$ & $(0.12)_{1}$ & 0.55 & 1.00 & 1.80 & 0.7 & 0.7 & 0.7 & -0.03 & -0.03 & -0.03 \\
\hline Summer and Fall, 2nd & $(0.60)_{1}$ & $(0.55)_{1,2}$ & $(0.27)_{2,3}$ & $(0.12)_{3}$ & 0.55 & 1.00 & 1.80 & 0.7 & 0.7 & 0.7 & -0.03 & -0.03 & -0.03 \\
\hline Fall, 1st & 0.60 & 0.55 & 0.27 & 0.12 & 0.55 & 1.00 & 1.80 & $(0.7)_{1}$ & $(0.7)_{2}$ & $(0.7)_{3}$ & $(-0.03)_{1}$ & $(-0.03)_{2}$ & $(-0.03)_{3}$ \\
\hline Fall, 2nd & 0.60 & 0.55 & 0.27 & 0.12 & 0.55 & 1.00 & 1.80 & $(0.7)_{1}$ & $(0.6)_{2}$ & $(0.75)_{3}$ & $(-0.03)_{1}$ & $(-0.03)_{2}$ & $(-0.03)_{3}$ \\
\hline Ext. Summer and Fall $1^{s t}$ & $(0.60)_{1}$ & $(0.55)_{2}$ & $(0.27)_{3}$ & 0.12 & 0.55 & 1.00 & 1.80 & 0.65 & 0.6 & 0.75 & $(-0.02)_{1}$ & $(-0.03)_{2}$ & $(-0.03)_{3}$ \\
\hline Ext. Summer and Fall $2^{\text {nd }}$ & $(0.70)_{1}$ & $(0.55)_{2}$ & $(0.27)_{3}$ & 0.12 & 0.55 & 1.00 & 1.80 & 0.65 & 0.6 & 0.75 & $(-0.025)_{1}$ & $(-0.03)_{2}$ & $(-0.03)_{3}$ \\
\hline Final Result & 0.70 & 0.55 & 0.27 & 0.12 & 0.55 & 1.00 & 1.80 & 0.65 & 0.6 & 0.75 & -0.025 & -0.025 & -0.03 \\
\hline
\end{tabular}

\subsection{Global minimization and sensitivity analysis}

While evaluating an initial approximation, we sought minima of the cost functions $J(\mathcal{C})$ measuring discrepancy over periods $\left\{\Delta_{k}\right\}$. In this subsection, we perform global minimization of the cost function with respect to all parameters in $\mathrm{C}$ over the entire period of measurements 22 July 2001 until 15 May 2002 used for calibration. Also, we analyze sensitivity of an initial approximation derived from minimizing the cost function globally with respect to all parameters.

In global minimization problems, a starting point from which iterations begin is given by the initial approximation evaluated in the previous subsection, see Table 3, the last row. As a result of global minimization problem, we obtain the parameters (thermal properties, porosity and coefficients specifying the unfrozen water content for each soil horizon) which can depend on values $t_{s}$ and $t_{e}$ determining the period over which discrepancy between observed and modeled temperatures is measured. In global minimization problems, the constant $t_{e}$ is associated with an end of "winter" interval during which the soil is completely frozen. But since, the soil is frozen for several months for a cold permafrost region, the cost function does not significantly depends on $t_{e}$ if $t_{e}$ varies within two week limits. However, the value of $t_{s}$ is associated with beginning of "summer and fall" interval during which the ground is thawed. Since, the ground is thawing during a relatively short period of time for cold permafrost regions, we consider several values of $t_{s}$ and minimize the cost function with respect to all parameters.

Results of minimization are listed in Table 4. It shows that the results of global minimization do not significantly depend on constants $t_{s}$, if the interval $\left[t_{s}, t_{e}\right]$ represents thawed and frozen states of the soil. Using averaged values of the thermal properties, we compute the temperature dynamics for the entire period of observations. Comparison of the calculated and measured temperatures at different depths and at time intervals used for calibration are shown in Figs. 10, 11 and 12. During the winter, the calculated temperature closely follows the observed temperature within the uncertainty of thermistor measurements. During the summer, the difference between the measured and calculated temperatures is larger but does not exceed $0.3^{\circ} \mathrm{C}$ for sensors in the mineral soil. This larger discrepancy between the measured and computed temperatures can be partially explained by over-simplifying physics and neglecting water dynamics in the upper organic horizons.

Finally, in order to show that the found initial approximation (the last row in Table 3) lies close to the true values of soil properties, we use it to compute the soil temperature dynamics through 22 February 2005 . Note that the time interval from 22 May 2002 until 22 February 2005 was not used to find the soil properties. In Fig. 13, we plot the measured and calculated temperature dynamics at $0.55 \mathrm{~m}$ depths. The plots with solid symbols mark temperature dynamics computed for the found initial approximation, and for the best guess values (in the middle of the variability range, shown in Table 1). We note that the guessed values are used to provide benchmark temperature dynamics against which we show effectiveness of our approach. The benchmark temperature is much warmer during summer, and the freeze-up occurs several days later than in the measured temperature. The benchmark temperature dynamics during winter closely follows the measured temperature dynamics, since the middle of the variability range, for which the benchmark temperature was computed, almost matches the found initial approximation. The difference between the measured temperature dynamics and the one calculated for the found initial approximation is typically less than $0.25^{\circ} \mathrm{C}$.

\section{Discussion and limitation of the proposed method}

We concentrate on finding soil thermal properties by minimizing the multivariate cost function $J$. There are many well known methods that find a minimum of $J$, including stochastic, heuristic and gradient type algorithms (Goldberg, 1989; Fletcher, 2000; Robert and Casella, 2004). Since we focus on a gradient type algorithm, a special care is necessary to 

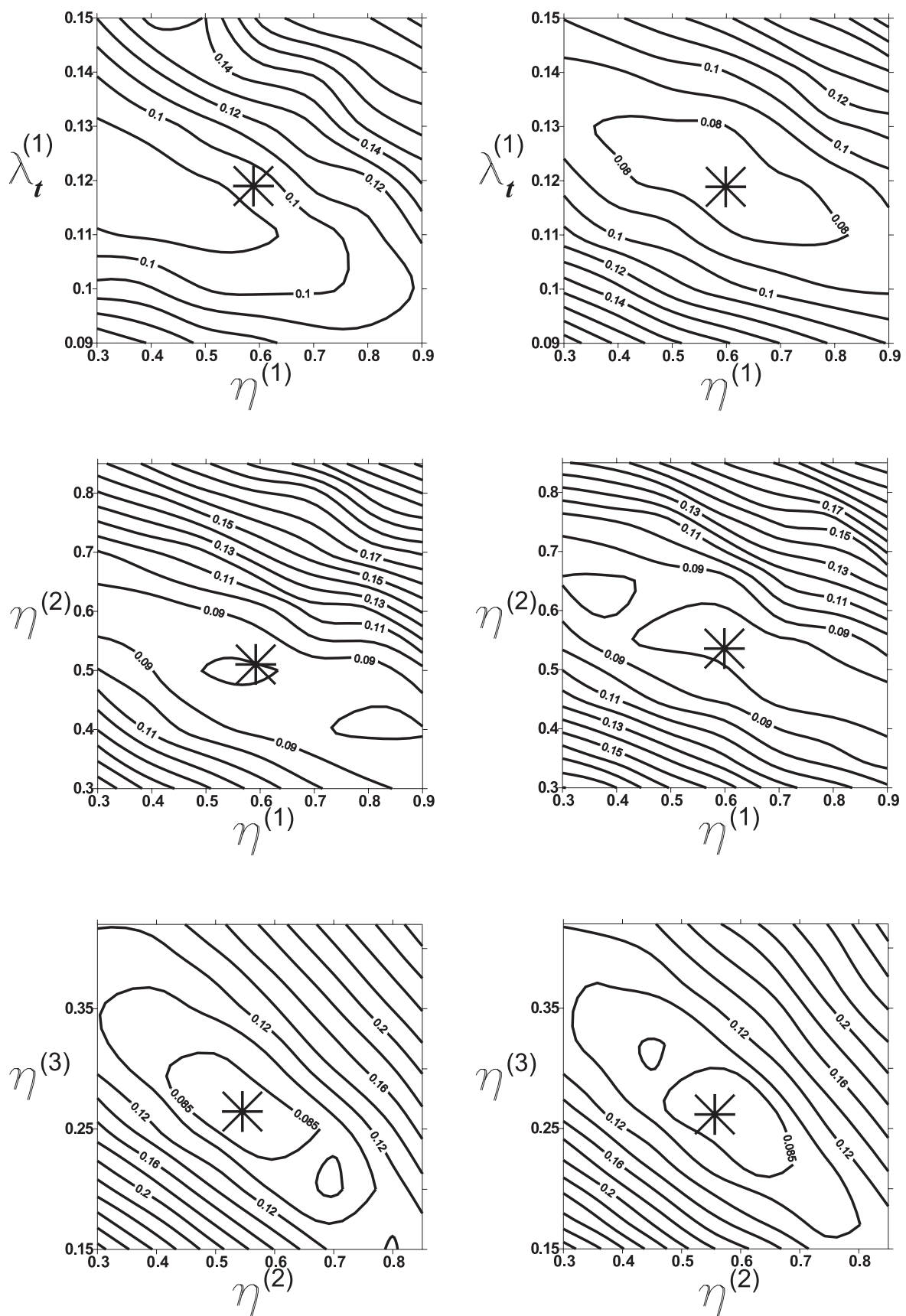

Fig. 9. Selection of the thermal conductivity $\lambda_{t}^{(1)}$ and the soil porosity $\eta^{(1)}, \eta^{(2)}, \eta^{(3)}$ by minimizing the cost function associated with the "summer and fall" interval. The left and right column are associated with the first and the second iterations, respectively. The stars mark selected values of parameters after completing the iteration. Note that at the second iteration stars and locations of all minima are coincide.

select the initial approximation to the soil properties. For example, if the gradient type algorithm is started outside the basin of attraction of the proper minimum, then due to existence of multiple local minima it can converge to physically non-realistic combination of parameters in $\mathcal{C}$. Stochastic and heuristic algorithms can possibly avoid the problem of select- ing the initial approximation, since they are not get trapped in a neighborhood of the local minimum. However, a quality control of soil properties recovered by either stochastic or heuristic algorithm arises. For example, it is feasible that several local minima can have the same value and correspond to substantially different combinations of parameters in the 
Table 4. Global minimization with respect to all parameters in the control. Each realization is specified by the time interval $\left[t_{s}, t_{e}\right]$ over which the discrepancy between the data and computed temperature dynamics is evaluated. In all case, the constant $t_{e}$ is 15 May 2002 .

\begin{tabular}{lccccccccccccc}
\hline \multirow{2}{*}{$t_{S}$} & $\eta^{(1)}$ & $\eta^{(2)}$ & $\eta^{(3)}$ & $\lambda_{t}^{(1)}$ & $\lambda_{f}^{(1)}$ & $\lambda_{f}^{(2)}$ & $\lambda_{f}^{(3)}$ & $b^{(1)}$ & $b^{(2)}$ & $b^{(3)}$ & $T_{*}^{(1)}$ & $T_{*}^{(2)}$ & $T_{*}^{(3)}$ \\
\hline August,18 & 0.703 & 0.560 & 0.272 & 0.120 & 0.562 & 0.983 & 1.797 & 0.655 & 0.596 & 0.750 & -0.0251 & -0.0253 & -0.0301 \\
August,22 & 0.721 & 0.557 & 0.272 & 0.122 & 0.559 & 0.973 & 1.809 & 0.673 & 0.558 & 0.757 & -0.0256 & -0.0249 & -0.0295 \\
August,26 & 0.718 & 0.546 & 0.272 & 0.122 & 0.559 & 0.962 & 1.801 & 0.657 & 0.597 & 0.755 & -0.0250 & -0.0251 & -0.0303 \\
August,30 & 0.712 & 0.549 & 0.272 & 0.121 & 0.556 & 0.967 & 1.801 & 0.655 & 0.601 & 0.755 & -0.0251 & -0.0251 & -0.0302 \\
September,3 & 0.712 & 0.544 & 0.274 & 0.123 & 0.559 & 0.980 & 1.816 & 0.665 & 0.551 & 0.750 & -0.0255 & -0.0255 & -0.0298 \\
September,7 & 0.718 & 0.534 & 0.274 & 0.123 & 0.560 & 0.966 & 1.789 & 0.660 & 0.603 & 0.747 & -0.0250 & -0.0252 & -0.0297 \\
\hline
\end{tabular}

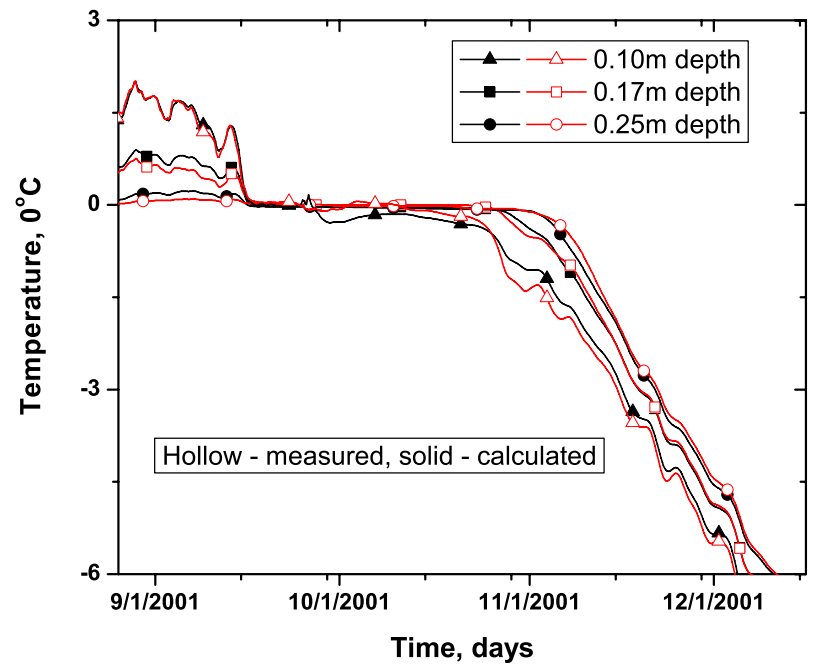

Fig. 10. Measured (hollow) and calculated (solid) temperature at $0.10,0.17$ and $0.25 \mathrm{~m}$ depth. The time interval is associated with the "summer and fall" period.

control vector $\mathcal{C}$. Thus, straight forward application of these algorithms can result in soil properties that are different from the physically realistic soil properties several fold.

In this article, we find an initial approximation to the thermal properties. This initial approximation can be later used in gradient type algorithm both as a starting point and a regularization. We admit that we find one of the possible realizations for the initial approximations. However, in the process of its computation, we obtain limiting boundaries on parameters in $\mathcal{C}$ which can constrain multivariate minimization, independent on the type of algorithm, i.e. stochastic, heuristic, or the gradient type.

We describe a technique to find an initial approximation to the thermal properties of soil horizons. This technique approximates the thermal conductivity, porosity, unfrozen water content curve in horizons where no direct temperature measurements are available. One of the limitations is that it requires values of heat capacities, since at certain time periods it is possible to estimate thermal diffusivity only but not thermal conductivity and heat capacity separately.

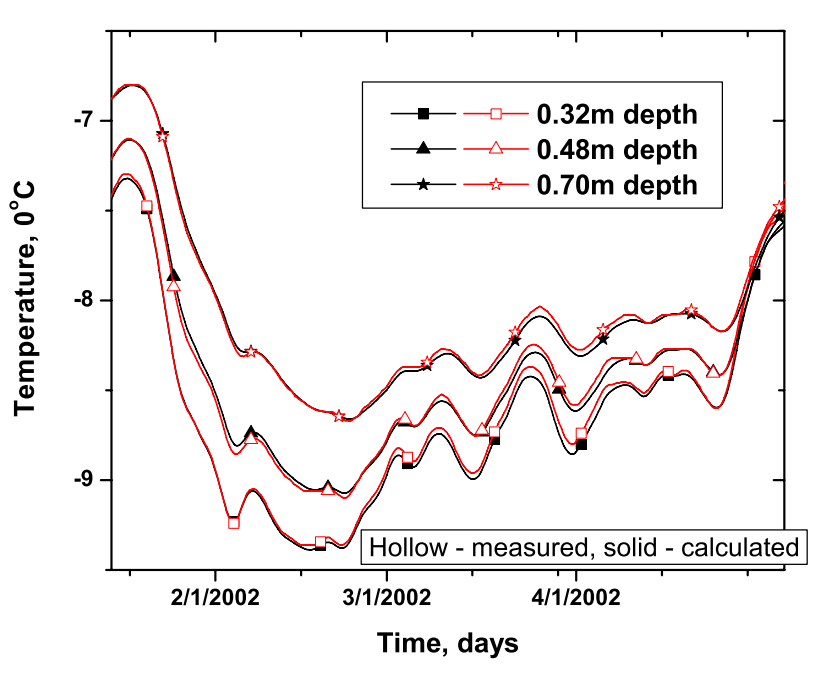

Fig. 11. Measured (hollow) and calculated (solid) temperature at $0.32,0.48$, and $0.70 \mathrm{~m}$ depth. The time interval is associated with the "winter" period.

Since, due to a short distance between points at which the upper and lower boundary conditions are specified, there is uncertainty in evaluation of the thermal conductivity for the frozen ground. This uncertainty is related to a progressive lag in the phase of the temperature wave, defined by the Second Fourier Law (Carslaw and Jaeger, 1959). The longer the lag, the better the thermal conductivity can be estimated. For the Happy Valley site, the temperature lag at $1 \mathrm{~m}$ depth is about 10 days. Our experience suggests that the 20-30 day time lag is adequate to estimate thermal conductivity robustly. In the case of shorter time lags, we advocate placing of a thermal conductivity sensor in the mineral soil horizon. The thermal conductivity sensor consists of a heating element and a thermocouple embedded in a needle. More information regarding the sensor can be found in (Thermal Logic, 2001) and in references therein. One of the limitations on usage of the thermal conductivity sensor is that it generates correct values of the thermal conductivity for thawed or completely frozen soil in which active phase change processes do not occur. 


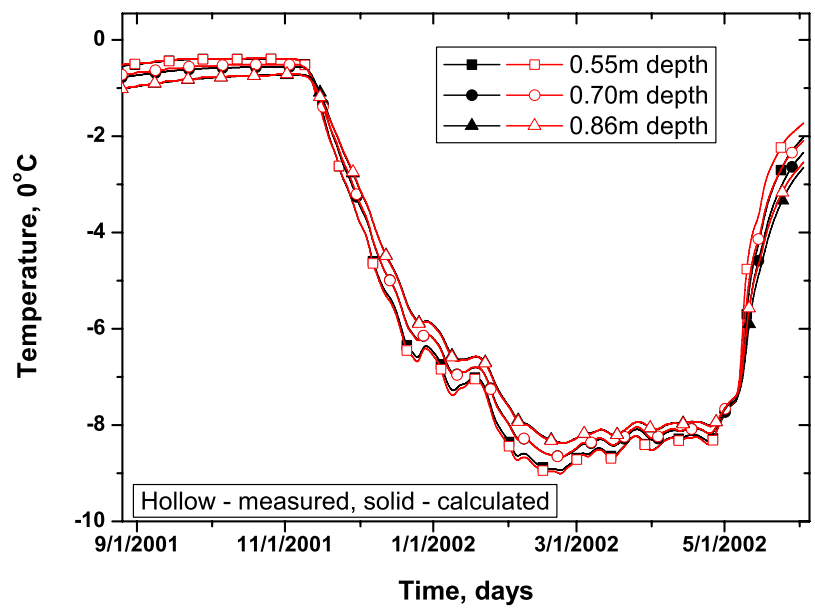

Fig. 12. Measured (hollow) and calculated (solid) temperature at $0.55,0.70$ and $0.86 \mathrm{~m}$ depth during the entire period of measurements used for calibration.

It should be noted that recovery of the thermal properties of the organic cover (e.g. moss layer) is given as an integrated approach in the following sense. Complex physical processes occurring in the organic cover that include nonconductive heat transfer (Kane et al., 2001) are taken into account by estimating some effective thermal properties which are constants for the entire season. We acknowledge that the estimated thermal properties of the organic layer could be different in nature, but we recover them in such a way that the temperature in the active layer and permafrost should correspond to the measured one.

In the proposed model we used 1-D assumption regarding the heat diffusion in the active layer, which sometimes is not applicable due to hummocky terrain in the Arctic tundra. Another assumption used in the model is that frost heave and thaw settlement is negligibly small and there is no ice lens formation in the ground during freezing. Therefore, the proposed method could be only applied where these assumption are satisfied.

The proposed method allows computation of a volumetric content $\eta$ of water which changes its phase during freezing or thawing. Water content of liquid water that is tightly bound to soil particles and is not changing its phase can not be estimated, see Fig. 1.

\section{Conclusions}

We present a technique to calculate an approximation to the soil thermal properties, porosity, and parametrization of the unfrozen water content in order to use it in gradient type iterative minimization methods both as a starting point and as a regularization. To compute the approximation, we minimize the multivariate cost function describing discrepancy

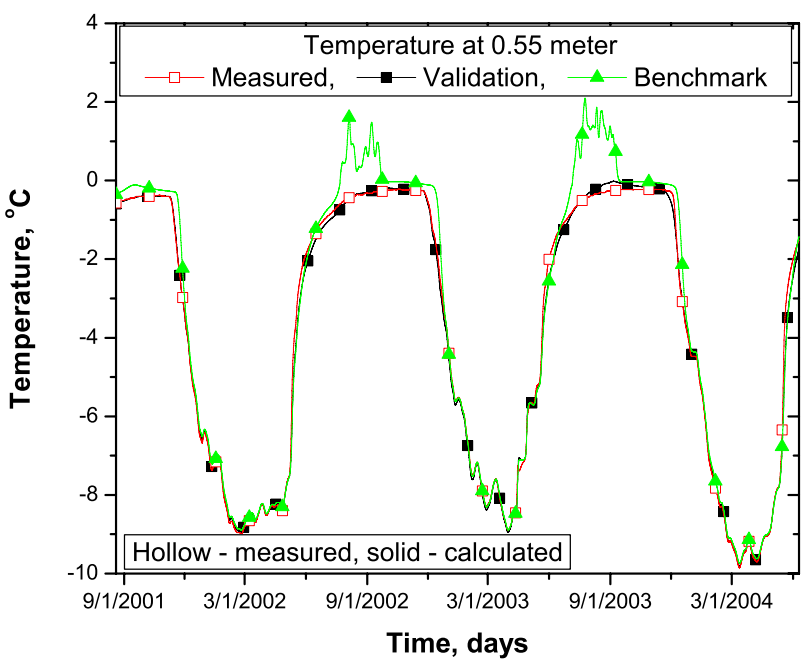

Fig. 13. Measured (hollow) and calculated (solid) temperature at $0.55 \mathrm{~m}$ depth during the entire period of measurements. The validation represents temperature dynamics computed for the found approximation to the soil thermal properties. The benchmark represents temperatures computed for the best guess soil properties, i.e. in the middle of ranges listed in Table 1.

between the measured and calculated temperatures over a certain time interval. We find the minimum by adopting a coordinate-wise iterative search technique to the specifics of our inverse problem. At each iteration, we select a particular set of soil properties and associate with them a certain time interval over which we minimize the cost function. After employing the proposed sequence of iterations, it is possible to find the approximation to all thermal properties and soil porosity.

Although there are several limitations to the proposed approach, we applied it to recover soil properties for Happy Valley site near Dalton highway in Alaska. The difference between the simulated and measured temperature dynamics over the periods of calibration is typically less than $0.3^{\circ} \mathrm{C}$. The difference between the simulated and measured temperatures over the consecutive time interval not used in calibration is less than $0.5^{\circ} \mathrm{C}$ which shows a good agreement with measurements, and validates that the found initial approximation lies close to the true values of soil properties.

In order to compute the cost function, it is necessary to calculate the soil temperature dynamics. Therefore, we developed a new finite element discretization of the Stefan-type problem on fixed coarse grids using enthalpy formulation. One of the advantages of the new method is that it allows computation of the temperature dynamics for the classical Stefan problem without any smoothing of the enthalpy. Also, new approach shows equal or better performance comparing to other finite element models of the ground thawing and freezing processes. 
Acknowledgements. We would like to thank J. Stroh, S. Marchenko, A. Kholodov, and P. Layer for all their advice, critique and reassurances along the way. We are thankful to reviewers and the editor for valuable suggestions making the manuscript easier to read and understand. This research was funded by ARCSS Program and by the Polar Earth Science Program, Office of Polar Programs, National Science Foundation (OPP-0120736, ARC-0632400, ARC-0520578, ARC-0612533, IARC-NSF CA: Project 3.1 Permafrost Research), by NASA Water and Energy Cycle grant, and by the State of Alaska.

Edited by: S. Gruber

\section{References}

ACIA: Impacts of a Warming Arctic: Arctic Climate Impact Assessment, Cambridge University Press, 139 pp., 2004.

Alifanov, O.: Inverse Heat Transfer Problems, Springer, Berlin, 348 pp., 1995.

Alifanov, O., Artyukhin, E., and Rumyantsev, S.: Extreme Methods for Solving Ill-Posed Problems with Application to Inverse Heat Transfer Problems, Begell House, New York, 306 pp., 1996.

Andersland, O. and Anderson, D.: Geotechnical Engineering for Cold Regions, McGraw-Hill, 566 pp., 1978.

Anderson, D. and Morgenstern, N.: Physics, chemistry and mechanics of frozen ground: a review, in: Proceedings of the 2nd International Conference on Permafrost, Yakutsk, USSR, 257288, 1973.

Avriel, M.: Nonlinear Programming: Analysis and Methods, Dover Publications, 554 pp., 2003.

Bazaraa, M., Sherali, H., and Shetty, C. M.: Nonlinear Programming: Theory and Algorithms, 2nd edn., John Wiley \& Sons, 1993.

Boike, J. and Roth, K.: Time domain reectometry as a field method for measuring water content and soil water electrical conductivity at a continuous permafrost site, Permafrost Periglac., 8, 359-370, 1997.

Brown, J., Ferrians, O., Heginbottom, J. J., and Melnikov, E.: Circum-Arctic map of permafrost and ground-ice conditions, U.S. Geological Survey Circum-Pacific Map CP-45, 1:10000 000, reston, Virginia, 1997.

Carslaw, H. and Jaeger, J.: Conduction of Heat in Solids, Oxford University Press, London, 520 pp., 1959.

Carson, J.: Analysis of soil and air temperatures by Fourier techniques, J. Geophys. Res., 68, 2217-2232, 1963.

Ciarlet, P.: The Finite Element Method for Elliptic Problems, North-Holland, Amsterdam, 530 pp., 2002.

Comini, G., Giudice, S. D., Lewis, R., and Zienkiewicz, O.: Finite element solution of non-linear heat conduction problems with special reference to phase change, Int. J. Numer. Meth. Eng., 8, 613-624, 1974.

Dalhuijsen, A. and Segal, A.: Comparison of finite element techniques for solidification problems, Int. J. Numer. Meth. Eng., 23, 1807-1829, 1986.

de Vries, D.: Physics of the Plant Environment, chap. Thermal properties of soils, edited by: van Wijk, W. R., Wiley, New York, 210-235, 1963.

Dennis, J. and Schnabel, R.: Numerical Methods for Unconstrained Optimization and Nonlinear Equations, Prentice-Hall, 394 pp.,
1987.

Fletcher, R.: Practical Methods of Optimization, John Wiley \& Sons, 450 pp., 2000.

Galushkin, Y.: Numerical simulation of permafrost evolution as a part of sedimentary basin modeling: permafrost in the PlioceneHolocene climate history of the Urengoy field in the West Siberian basin, Can. J. Earth Sci., 34, 935-948, 1997.

Goldberg, D.: Genetic Algorithms in Search, Optimization, and Machine Learning, Addison Wesley, 432 pp., 1989.

Goodrich, W.: The influence of snow cover on the ground thermal regime, Can. Geotech. J., 19, 421-432, 1982.

Gupta, S.: The Classical Stefan Problem, Elsevier, Amsterdam, 404 pp., 2003.

Hinkel, K.: Estimating seasonal values of thermal diffusivity in thawed and frozen soils using temperature time series, Cold Reg. Sci. Technol., 26, 1-15, 1997.

Hinzman, L., Kane, D., Gleck, R., and Everett, K.: Hydrological and thermal properties of the active layer in the Alaskan Arctic, Cold Reg. Sci. Technol., 19, 95-110, 1991.

Hobbs, P.: Ice Physics, Claredon Press, Oxford, 856 pp., 1974.

Hurley, S. and Wiltshire, R. J.: Computing thermal diffusivity from soil temperature measurements, Computers and Geosciences, 19, 475-477, 1993.

Jaeger, J. C. and Sass, J. H.: A line source method for measuring the thermal conductivity and diffusivity of cylindrical specimens of rock and other poor conductors, J. Appl. Phys., 15, 1187-1194, 1964.

Javierre, E., Vuik, C., Vermolen, F., and van der Zwaag, S.: A comparison of numerical models for one-dimensional Stefan problems, J. Comput. Appl. Math., 192, 445-459, 2006.

Kane, D., Hinzman, L., and Zarling, J.: Thermal response of the active layer in a permafrost environment to climatic warming, Cold Reg. Sci. Technol., 19, 111-122, 1991.

Kane, D., Hinkel, K., Goering, D., Hinzman, L., and Outcalt, S.: Non-conductive heat transfer associated with frozen soils, Global Planet. Change, 29, 275-292, 2001.

Kolmogorov, A. and Fomin, S.: Introductory Real Analysis, Prentice-Hall, New York, 403 pp., 1975.

Lagarias, J., Reeds, J., Wright, M., and Wright, P.: Convergence properties of the Nelder-Mead simplex method in low dimension, SIAM J. Optimiz., 9, 112-147, 1998.

Lemmon, E.: Numerical Methods in Thermal Problems, chap. Phase change technique for finite element conduction code, edited by: Lewis, R. W. and Morgan, K., Pineridge Press, Swansea, UK, 149-158, 1979.

Ling, F. and Zhang, T.: Impact of the timing and duration of seasonal snow cover on the active layer and permafrost in the Alaskan Arctic, Permafrost Periglac., 14, 141-150, 2003.

Lovell, C.: Temperature effects on phase composition and strength of partially frozen soil, Highway Research Board Bulletin, 168, 74-95, 1957.

Lunardini, V.: Freezing of soil with an unfrozen water content and variable thermal properties, CRREL Report 88-2, US Army Cold Regions Research and Engineering Lab, 30 pp., 1987.

Mölders, N. and Romanovsky, V.: Long-term evaluation of HTSVS frozen ground/permafrost component using observations at Barrow, Alaska., J. Geophys. Res., 111, D04105, doi:10.1029/2005JD005957, 2006. 
Thermal Logic: Thermal conductivity sensor user's manual, PO Box 781, Pullman, WA, 99163, 3 edn., 25 pp., 2001.

McGaw, R. W., Outcalt, S. I., and Ng, E.: Thermal properties of wet tundra soils at Barrow, Alaska, in: Proceedings of 3rd International Conference on Permafrost, vol. 1, National Research Council of Canada, Ottawa, Canada, 47-53, 1978.

Morgan, K., Lewis, R., and Zienkiewicz, O.: An improved algorithm for heat conduction problems with phase change, Int. J. Numer. Meth. Eng., 12, 1191-1195, 1978.

Mundim, M. and Fortes, M.: Numerical Methods in Thermal Problems, vol. 6, chap. Evaluation of finite element method utilized in the solution of solid-liquid phase change problems, edited by: Lewis, R. W. and Morgan, K., Pineridge Press, Swansea, UK, 90-100, 1979.

Nelson, F. and Outcalt, S.: Anthropogenic geomorphology in northern Alaska, Polar Geography, 3, 17-48, 1987.

Oleson, K., Dai, Y., Bonan, G., Bosilovich, M., Dickinson, R., Dirmeyer, P., Hoffman, F., Houser, P., Levis, S., Niu, G.-Y., Thornton, P., Vertenstein, M., Yang, Z.-L., and Zeng, X.: Technical description of the Community Land Model (CLM), NCAR tech. note NCAR/TN-461+STR, NCAR, 173 pp., 2004.

Osterkamp, T. and Romanovsky, V.: Characteristics of changing permafrost temperatures in the Alaskan Arctic, USA, Arctic Alpine Res., 28, 267-273, 1996.

Osterkamp, T. and Romanovsky, V.: Freezing of the active layer on the coastal plain of the Alaskan Arctic, Permafrost Periglac., 8, 23-44, 1997.

Permyakov, P.: Methods of determining the characteristics of dispersed media at a phase transition, Russian Physics Journal, 47(3), 240-246, 2004.

Pham, Q.: Comparison of general-purpose finite element methods for the Stefan problem, Numer. Heat Tr. B-Fund., 27, 417-435, 1995.

Pinder, G. and Gray, W.: Finite Element Simulation in Surface and Subsurface Hydrology, Academic Press, New York, 295 pp., 1977.

Rank, E., Katz, C., and Werner, H.: On the importance of the discrete maximum principle in transient analysis using finite element methods, Int. J. Numer. Meth. Eng., 19, 1771-1782, 1983.

Robert, C. and Casella, G.: Monte Carlo Statistical Methods, Springer-Verlag, 645 pp., 2004.

Romanovsky, V. and Osterkamp, T.: Interannual variations of the thermal regime of the active layer and near-surface permafrost in Northern Alaska, Permafrost Periglac., 6, 313-335, 1995.

Romanovsky, V. and Osterkamp, T.: Thawing of the active layer on the coastal plain of the Alaskan Arctic, Permafrost Periglac., 8, $1-22,1997$.

Romanovsky, V. and Osterkamp, T.: Effects of unfrozen water on heat and mass transport processes in the active layer and permafrost, Permafrost Periglac., 11, 219-239, 2000.

Samarskii, A. and Vabishchevich, P.: Computational Heat Transfer, Mathematical Modeling, vol. 1, Wiley, 418 pp., 1996.

Sass, J., Lachenbruch, A., and Munroe, R.: Thermal conductivity of rocks from measurements on fragments and its application to heat-flow determinations, J. Geophys. Res., 76, 3391-3401, 1971.
Sazonova, T., Romanovsky, V., Walsh, J., and Sergueev, D.: Permafrost dynamics in the 20th and 21st centuries along the East Siberian transect, J. Geophys. Res., 109, D01108, doi:10.1029/2003JD003680, 2004.

Schmugge, T., Jackson, T., and McKim, H.: Survey of methods for soil moisture determination, Water Resour. Res., 16, 961-979, 1980.

Smith, M. and Tice, A.: Measurement of the unfrozen water content of soilscomparison of NMR and TDR methods, CRREL Report 88-18, US Army Cold Regions Research and Engineering Lab, 16 pp., 1988.

Stafford, J.: Remote, non-contact and in-situ measurement of soil moisture content: a review, J. Agr. Eng. Res., 14, 151-172, 1988.

Thacker, W.: The role of the Hessian matrix in fitting models to measurements, J. Geophys. Res., 94, 6177-6196, 1989.

Thacker, W. and Long, R.: Fitting dynamics to data, J. Geophys. Res., 93, 1227-1240, 1988.

Tice, A., Oliphant, J., Nakano, Y., and Jenkins, T.: Relationship between the ice and unfrozen water phases in frozen soil as determined by pulsed nuclear magnetic resonance and physical desorption data, CRREL Report 82-15, US Army Cold Regions Research and Engineering Lab, 12 pp., 1982.

Tikhonov, A. and Samarskii, A.: Equations of Mathematical Physics, Pergamon, 776 pp., 1963.

Tikhonov, A., Leonov, A., and Yagola, A. G.: Nonlinear Ill-Posed Problems, Chapman and Hall, London, 392 pp., 1996.

Topp, G. C., Davis, J. L., and Annan, A. P.: Electromagnetic determination of soil water content: measurements in coaxial transmission lines, Water Resour. Res., 16, 574-582, 1980.

Ulaby, F., Moore, R., and Fung, A.: Microwave Remote Sensing, Addison-Wesley, Reading, MA, Vol. II, 1064 pp., 1982.

Voller, V. and Swaminathan, C.: Fixed grid techniques for phase change problems: a review, Int. J. Numer. Meth. Eng., 30, 875$898,1990$.

Watanabe, K. and Mizoguchi, M.: Amount of unfrozen water in frozen porous media saturated with solution, Cold Reg. Sci. Technol., 34, 103-110, 2002.

Williams, P.: Properties and behaviour of freezing soils, Tech Rep. 72, Norwegian Geotechnical Institute, 120 pp., 1967.

Yershov, E.: General Geocryology, Cambridge University Press, Cambridge, 604 pp., 1998.

Yoshikawa, K., Overduin, P., and Harden, J.: Moisture content measurements of moss (Sphagnum spp.) using commercial sensors, Permafrost Periglac., 15, 309-318, 2004.

Zhang, T. and Osterkamp, T. E.: Considerations in determining the thermal diffusivity from temperature time series using finite difference methods, Cold Reg. Sci. Technol., 23, 333-341, 1995.

Zhuang, Q., Romanovsky, V., and McGuire, A.: Incorporation of a permafrost model into a large-scale ecosystem model: evaluation of temporal and spatial scaling issues in simulating soil thermal dynamics, J. Geophys. Res., 106, 33 649-33 670, 2001.

Zienkiewicz, O. and Taylor, R.: The Finite Element Method, vol. 1, McGraw-Hill, London, 470 pp., 1991. 